\title{
EXAMINING DEMOGRAPHIC CHARACTERISTICS AND SETTLEMENT PATTERNS OF ETHNOCULTURALLY-DIVERSE SPECIFIC LONG-STANDING AND RECENT OLDER IMMIGRANTS IN THE TORONTO CMA, 2016
}

\author{
by \\ Adam Anthony \\ Bachelor of Arts, Ryerson University 2015
}

A Major Research Paper

presented to Ryerson University

\author{
in partial fulfillment of the \\ requirements for the degree of \\ Master of Spatial Analysis \\ in the program of \\ Spatial Analysis
}

Toronto, Ontario, Canada, 2019

(C) Adam Anthony, 2019 


\section{Author's Declaration}

AUTHOR'S DECLARATION FOR ELECTRONIC SUBMISSION OF A MRP

I hereby declare that I am the sole author of this MRP. This is a true copy of the MRP, including any required final revisions.

I authorize Ryerson University to lend this MRP to other institutions or individuals for the purpose of scholarly research.

I further authorize Ryerson University to reproduce this MRP by photocopying or by other means, in total or in part, at the request of other institutions or individuals for the purpose of scholarly research.

I understand that my MRP may be made electronically available to the public. 
Examining Demographic Characteristics and Settlement Patterns of Ethnoculturally-specific Longstanding and Recent Older Immigrants in The Toronto CMA, 2016

2019

Adam Anthony

Master of Spatial Analysis

Ryerson University

\begin{abstract}
Understanding population characteristics and residential patterns of recent and long-standing older immigrants is important to ensure that settlement services are adequately supporting a diverse and vulnerable population. This research paper represents a pilot study to fill in the gap found in the already limited scholarship on the characterization, spatial distribution and in-group differences of older immigrants in the Toronto CMA. Firstly, it explores the nuanced differences in population composition of four ethnocultural-specific subgroups representing long-standing (Italian and Portuguese) and recent immigrants (Chinese and South Asian) and secondly, it identifies clusters of recent immigrants that are settling outside of the long-standing ethnocultural enclaves. Despite having higher rates of education than their long-standing counterparts, Chinese and South Asian are characterized by low income prevalence and lack of knowledge of an official language. Hence, determining the multilingual composition of the South Asian and Chinese subgroups can facilitate language-specific settlement services within recent older South Asian and Chinese immigrant clusters.
\end{abstract}

Key words: older adults, immigration studies, recent immigrants, settlement challenges, low income, hot spot analysis, Toronto Census Metropolitan Area 


\section{Acknowledgements}

I would like to give special thanks to my supervisor, Dr. Lu Wang, for her guidance and encouragement throughout this research processes. To my friends, family and coworkers, thanks for cheering me on throughout this journey. Thanks to Max Piorkowski for taking care of the household duties over the past two years and to Tony Conte for being just the best boss I could have asked for. Thanks to Hallie Anthony for convincing me it will get better. Lastly, a special thanks to my plant babies for giving me the oxygen I needed to pull through and to Pickle for being so darn cute. 
Author's Declaration

Table of Contents

Abstract

Acknowledgements

List of Tables

List of Figures

1. Introduction

1.1 Background

1.2. Research questions

2. Literature review $\quad 5$

2.1 Settling in Canada as an older immigrant $\quad 5$

2.1.1 Income inequity for older immigrants $\quad 6$

2.1.2 Devaluation of education and limited employment 7

2.1.3 Ethnocultural and linguistic diversity amongst older immigrants 7

2.1.4 Accessible information and services $\quad 9$

2.2 Spatial distribution of immigrants in Toronto CMA 9

2.3 Summary of literature review 10

3. Data $\quad 11$

3.1 Study area and study population $\quad 11$

3.2 Demographic Data 12

4. Methods $\quad 16$

4.1 Cross-tabulations and line graphs based on custom census 17

4.2 Spatial Methods 17

5. Results $\quad 19$

5.1 Comparing older immigrants and older non-immigrant 19

5.1.1 Variation in socioeconomic and demographic composition 19

5.1.2 Variation in settlement patterns of older immigrants and older non-immigrants 23

5.1.3 Variation in settlement patterns of long-standing and recent older immigrants 26

5.2 Group-specific socioeconomic, demographic dimensions and settlement patterns 29

5.2.1 General variation in select socioeconomic and demographic dimensions 29

5.2.2 Education, landing period and language's influence on income 32

5.2.3 Group-specific settlement patterns

5.2.4 Variation in group-specific settlement patterns for long-standing and recent subgroups 38

6. Discussion and Conclusions $\quad 41$

6.1 Summary of findings $\quad 41$

6.2 Discussion of results $\quad 42$

6.2.1 Variation in socioeconomic and demographic composition 44

6.2.2 Variation in settlement patterns of long-standing and recent older immigrants 45

6.3 Implications and recommendations 48

6.4 Contribution to knowledge $\quad 49$

6.5 Limitations and future research $\quad 49$

$\begin{array}{ll}\text { References } & 52\end{array}$ 


\section{List of Tables}

Table 1: Data sources $\quad 11$

Table 2: Variables used in research paper $\quad 15$

Table 3: Socioeconomic and demographic dimensions of older immigrant and non-immigrants 20

Table 4: Detailed group-specific socioeconomic and demographic population compositions 30

Table 5: Share of each older immigrant ethnocultural subgroup by long-standing or recent 35 categorization 


\section{List of Figures}

Figure 1: Proportion of population per Census Tract that are older immigrants, 2016

Figure 2: Ethnocultural composition of Toronto Census Metropolitan Area's older immigrant

population

Figure 3: Comparing income of older immigrants and older non-immigrants based on education

Figure 4: Proportion and hot spots of older immigrants and older non-immigrants

Figure 5: Settlement pattern hot spots for long-standing and recent older immigrants

Figure 6: Hot spot of older immigrant settlement during five immigration periods

Figure 7: Income distribution for Chinese, Italian, Portuguese and South Asian older immigrants

Figure 8: Education distribution for Chinese, Italian, Portuguese and South Asian older

immigrants

Figure 9: Income distribution based on secondary variable for each ethnocultural subgroup

Figure 10: Location Quotient of older immigrant subgroups

Figure 11: Long-standing and recent Chinese older immigrant settlement hot spots

Figure 12: Long-standing and recent Italian older immigrant settlement hot spots

Figure 13: Long-standing and recent South Asian older immigrant settlement hot spots 


\section{Introduction}

\subsection{Background}

The trend in greying populations is a global concern. The World Health Organization's National Institute on Aging (2011) projects that between 2011 and 2050 the number of people aged 65 or above will increase from $8 \%$ to $16 \%$ globally, amounting to a staggering 1.5 billion older adults around the world. Particularly, in one of the most populous countries, China's older population will triple over the next three decades, with an estimated 100 million Chinese individuals 80 years old or above (National Institute on Aging, 2011). This dramatic increase in volume and proportion of older adults is further compounded by the increased average life expectancy observed over the past 100 years at a global scale (National Institute on Aging, 2011). This shifting population structure is evidently reflected in Canada's population composition, such that in 2016, 17\% of Canada's population were 65 years and above, from 15\% in 2011 and $8 \%$ in 1996. By 2036, this cohort is projected to comprise almost a quarter of the nation's population (Chui, Tran \& Maheux, 2007; Ng, 2012; Ng, Sanmartin, Tu \& Manuel, 2015; Statistics Canada, 2012b). Moreover, Canada's median age has also seen an incline and is expected to increase to 44 years older by 2036, up from 40 in 2009 (Edmonston, 2016). Simultaneous to an aging demographic, Canada's population is diversifying and composed largely of immigrants and their descendants (Edmonston, 2016)

Increasing levels of immigration have been observed in Canada, and most recently the immigrant population grew from $19.8 \%$ in 2006, to $20.6 \%$ in 2011, then to $21.4 \%$ in 2016 (Statistics Canada, 2013). This national increase is amplified in Census Metropolitan Areas, where Toronto, Montreal and Vancouver CMAs are the most attractive immigrant-receiving destinations in Canada, thus receiving the highest volume of immigrants (Statistics Canada, 2007). The Toronto CMA, as the most popular destination, observed immigrant population growth of $45.7 \%$ in 2006 , to $46 \%$ in 2011 , then to $46.3 \%$ in 2016 (Statistics Canada, 2016, 2018b). Linking Canada's greying population and the immigrant population growth is that immigrants hold the highest share of the population 65 or above in the Toronto CMA, where a staggering 71.3\% of older adults in 2016 are immigrants up from 67\% in 2006 (Ng, 2012; Statistics Canada, 2011; Turcotte, 2007). Net population growth as the result of immigration will echo this increase, where by $203682 \%$ of Canada's annual population growth will be due to net migration, up from 58\% in 2009 (Edmonston, 2016). As populations are aging and immigration is on the rise, the Toronto CMA is also experiencing a shift in immigrant sending-countries, resulting in a diversification of newcomers not seen before. According to the 2011 Census, smaller proportions of immigrants are coming from European nations, while over half of all immigrants (56.9\%) are arriving from Asian countries (Statistics Canada, 2013). The resulting diversification of immigrant ethnocultures, inherently pressurizes and influences the demand, quality and accessibility of culturally appropriate social, financial and 
settlement services, particularly for older immigrants (Chui et al., 2007; Malenfant, Lebel \& Martel, 2010).

Adjustment and integration into Canadian society is challenging for immigrants, but particularly compounded when the immigrant demographic is older and ethnoculturally diverse. Encountering settlement challenges can depress an older immigrant's economic outcomes and amplify the degree of severity imposed upon their arrival. Period of immigration is an additional factor that can compound settlement challenges. After landing, immigrants encounter barriers to integration and adjustment such as the devaluation of language, culture and education, lack of understanding of Canada's official languages, vulnerability to chronic low income, and an inability to access adequate governmental support programs, most importantly, employment-related settlement services (Creese, 2007; Dempsey, 2005; Galabuzi, 2005; Lo and Wang, 2004; Mandell, Borras \& Phonepraseuth, 2018). Unfortunately, these settlement challenges are exacerbated through societal perceptions of immigrants as a homogenous group of people with similar needs, regardless of ethnocultural origin. This problematic perception fails to acknowledge or seeks to address the unique and nuanced barriers that older recent immigrants from different ethnocultural background might encounter during their settlement process (Laher, 2017; Lo \& Wang, 2004).

To provide context to this research paper an overview of Canada's immigration policies is provided. Understanding Canadian immigration history is critical in order to value Canada's current ethnocultural diversity and contribution immigration has had on Canada's population growth (Edmonston, 2016). Laws and regulations that govern the admission of immigrants in Canada have been established and evolved since 1869 with unfortunate consequences of discrimination based on migrants ethnicity and race (Dirks, 2006; Trebilcock, 1998; Van Dyk, 2019). Thankfully, immigration policy saw a shift in the past century which focused on skilled work experience and education as the main admission criteria (Van Dyk, 2019). Relevant immigration policies such as the Chinese Immigration Act, 1885 and the Royal Commission on Italian Immigration, 1904 were exclusionary legislations based on ethnocultural origin as the result of corrupt and exploitative homegrown foreign-labour structures (Trebilcock, 1998; Van Dyk, 2019). In 1906, the Immigration Act was introduced and was more restrictive on admission criteria and deportation policies, which was problematized as governing bodies could restrict entry based on ethnocultural origins, culture or race (Edmonston, 2016; Van Dyk, 2019). Subsequently, Canada experienced large waves of immigration from Eastern European sending-countries prior to World War One, and from Southern European sending-countries, in particular, Italian, preceding World War Two (Trebilcock, 1998). In response to post-war economic depression and disruptions of the labour market, the Immigration Act Amendment of 1991 was introduced, although it was revised, it still allowed the government to prohibit individuals from entering Canada based on ethnocultural background, race and class (Chui, 2016; Van Dyk, 2019). In 1923, the Chinese Immigration Act was called to restrict 
all Chinese immigration into the country by amending the criteria for admissions (Kaushal, 2015; Van Dyk, 2019). Between 1928 and 1971, Pier 21 in Halifax, Nova Scotia, played an important role in immigration of Italian and Portuguese immigrants as the port received upwards of 470,000 Italian and Portuguese immigrants (Dirks, 2006). Admissions criteria then began to pivot through The White Paper on Immigration, from 1966, which was a policy document that recommended a shift in criteria towards the recruitment of highly educated and skilled immigrants (Kaushal, 2015; Tannock, 2011; Van Dyk, 2019). In the subsequent year, Immigration Regulations, 1967, was introduced which established new and objective admissions criteria using a points system on categories like education, employment, age, national language proficiency and behaviour (Van Dyk, 2019). Post-1967 immigration policy gave way to see more racialized immigrants, from developing nations flock to Canada, as controls for discrimination on the basis of ethnicity and race were amended through the points system (Kaushal, 2015; Tannock, 2011; Van Dyk, 2019). In 1976, the Immigration Act was revised to include four new immigrant categories which promoted further immigration. Family-class immigrants are individuals that have a relative currently living in Canada, economic immigrants who are skilled in labour, refugees who are escaping persecution in their home nation and humanitarian who are given entry based on humanitarian or compassionate reasons (Sweetman, 2013; Beaujot, 2003; Ley, 2003; Wright, 1993) . Immigration streams have evolved since 1869 and it is evident that immigration might be the defining feature in the population growth of Canada (Edmonston, 2016). As the demography, ethnocultural diversity and growth of Canada has been largely influenced by immigration it should facilitate in-depth, critical and policy-informing scholarly research to better the outcomes of immigration settlement (Edmonston, 2016).

The purpose of this research paper is to demonstrate that long-standing and recent immigrant groups from four ethnoculturally-specific subgroups have greatly different settlement experiences once landed in the Toronto CMA. As age at immigration is a significant element to immigration adjustment and integration, it's critical to understand that age, ethnocultural origin and period of immigration might influence an immigrant's navigation through education and labour streams and through family development and income generation (Edmonston, 2016). In response to a scholarly call-to-action from Mandell et al. (2018), this research seeks to document both the socioeconomic experiences of older immigrants (recent and long-standing) by understanding the spatiality of their settlement. The study yields important implications for decision-makers to understand the detailed variations in settlement needs for older immigrants of vastly different backgrounds and landing periods.

\subsection{Research questions}

In this research paper, population characteristics and residential patterns of older immigrants and older non-immigrants in the Toronto CMA are assessed for differences and similarities relating to select 
socioeconomic and ethnocultural dimensions and spatial distribution. Additionally, socioeconomic and ethnocultural characteristics of the four largest ethnocultural immigrant groups in the Toronto CMA are examined. These groups are older immigrant Chinese, Italian, Portuguese and South Asian individuals. These four ethnocultural groups were selected as they represent almost $50 \%$ of the ethnocultural diversity for older immigrants in the Toronto CMA (Statistics Canada, 2018d). They can be grouped into two subgroups based on proportionately high immigration in two time periods. One being long-standing older immigrants, arriving before 2000 comprised of the Italian and Portuguese older immigrants. The second being recent older immigrants, arriving after 2001, comprised of Chinese and South Asian older immigrants. Four research questions were developed to evaluate these subgroups:

1. Do older immigrant and older non-immigrant populations differ in terms of socioeconomic (age, income, education, and gender) and ethnocultural characteristics (language spoken at home? and ethnicity)?

2. How does the settlement pattern of older immigrants and older non-immigrants vary spatially in the Toronto CMA and over time, from pre-1981 to 2016 ?

3. What are the socioeconomic (age, income, education, gender) and ethnocultural characteristics (language spoken, period of immigration) of the Chinese, Italian, Portuguese and South Asian older immigrant subgroups?

4. What are the residential patterns of the four ethnocultural subgroups across the CMA and what are the spatial and socioeconomic differences between recent (Chinese and South Asian) and long-standing (Italian and Portuguese) older immigrant subgroups?

This analysis represents a pilot study to fill in the gap found in the already limited scholarship on the characterization, spatial distribution and in-group differences of older immigrants in the Toronto CMA. This study will improve our understanding of how socioeconomic status might be influenced by group-specific characteristics including ethnocultural origin and period of immigration of older immigrants in the Toronto CMA. The relevant literature suggests that recent immigrants generally have a lower socioeconomic status than their long-standing counterparts (Aging in poverty in Canada, 2005; Dempsey, 2005; Government of Canada, 2018; Lo \& Wang, 2004; Mandell et al., 2018; Mental Health Commission of Canada, 2019; Ng, 2012; Picot \& Lu, 2017; Um \& Lightman, 2016) and that different immigrant communities tend to concentrate in different neighbourhoods to form spatial clusters (Hiebert, 2015; McDonald, 2004; Murdie, 2008; Simich, 2000; Vezina \& Houle, 2017). Understanding whether settlement challenges impact these subgroups differently, and most importantly, where these subgroups 
cluster in different neighbourhoods, can be used to identify systemic gaps in social and governmental services. The study yields important implications for decision-makers to understand the detailed variations in settlement needs for older immigrants of vastly different backgrounds and landing periods. Therefore, it facilitates the consideration and development of data-driven policy recommendations which aim to lessen the settlement obstacles encountered by the already vulnerable older immigrant communities.

\section{Literature review}

The goal of this literature review is to assess the relevant literature on the socioeconomic, ethnocultural and settlement patterns of immigrant populations, older population and older immigrants in Canada. Although older adults are a rapidly growing population cohort in Canada and almost three-quarters of the Toronto CMA's older adults are immigrants, there is limited scholarship on the in-group difference in settlement experiences for older long-standing and recent immigrants from different ethnocultural backgrounds (Ng, Lai, Rudner \& Orpana, 2012).

\subsection{Settling in Canada as an older immigrant}

Immigrants in the Toronto CMA hold the highest share of the older population (those aged 65 and above), composing 71.3\% of the older population in 2016, up from 67\% in 2006 (Ng, 2012; Statistics Canada, 2011; Turcotte, 2007). Of all older immigrants, $87.2 \%$ are long-standing residents of the Toronto CMA, arriving before 2000 , in stark contrast to almost $13 \%$ of older immigrants arriving after 2001, settling and aging as older adult newcomers (Statistics Canada, 2016b). In 2016, slightly less than 50\% of older immigrant populations hailed from only four ethnocultural regions globally: Chinese, Italian, Portuguese and South Asian. Of these four immigrant groups, Chinese and South Asian origins had the highest share of older immigrant newcomers arriving since 2011 (Statistics Canada, 2016a).

Immigration into Canada has largely been influenced by policy changes and global events since the 1800 s, which have created a unique set of circumstances to affect population migration (Lo \& Wang, 2004). Humanitarian concerns were a catalyst to this global trend spiking in the 1900s (Statistics Canada, 2016b). These major geopolitical events pushed individuals from different nations out of their home country at different periods throughout history. In an article investigating the convergence of Toronto's Chinese immigrant population, Lo and Wang (2004) outlined a chronology of events that have been found to influence the immigration of Chinese immigrants to Canada. Geopolitical events, such as world wars, civil wars, cultural revolutions, political turmoil and the development of new immigration policies were observed to have influenced immigration (Lo \& Wang, 2004). But these patterns are not unique to those of Chinese origins. In general, Canada has experienced waves of immigration from numerous sending- 
countries over the course of history. European migrants arrived in large numbers 150 years ago, originating from Great Britain, Germany and France, as well as China and Japan, followed by a second large wave of Eastern Europeans and Scandinavians in the early 1900s, and post-World War II migration from Great Britain, Western and Southern Europe (Statistics Canada, 2016b). A third wave of Eastern Europeans landed in the 1990s (Statistics Canada, 2016b). From the mid-19th century to the 2000s, the sending-countries shifted away from predominantly European nations, and towards countries from Asia, and central and South America. Assorted political events influenced mass exodus from countries such as Vietnam, Cambodia, Laos, Bermuda, Jamaica, Haiti, Trinidad and Tobago, China, India, and the Philippines (Statistics Canada, 2016b). The 2011 National Household Survey reported that Asian and African nations are the predominant sending-countries from which Canada receives immigrants from (Statistics Canada, 2016b).

Understanding the period in which an immigrant or immigrant group arrived to Canada is a necessary element to determine long-standing versus recent immigration patterns. Some scholars (Elgersma, 2007; Dempsey, 2005) categorize immigrants based on landing period, where established immigrants are those who arrived 17 or more years prior to the current year. They also classified shortterm immigrants as those who arrived seven to 16 years prior to the current year, and recent immigrants as those who arrived one to six years prior to the current year. Their analysis included a variable on age at immigration. A publication from 2018 produced by the multiple Statistics Canada departments defines a recent immigrant as a foreign-born individual that migrated to Canada within the past five years, therefore an established immigrant would be one that arrived over five years from the period of analysis (Chui, Flanders \& Anderson, 2018). However, given that the Canadian Census is conducted every five years, it might be beneficial to amend the aforementioned landing period classes in future research.

\subsubsection{Income inequity for older immigrants}

It is well documented that chronic low income is experienced by older immigrants, specifically older recent immigrants in Canada (Aging in poverty in Canada, 2005; Dempsey, 2005; Government of Canada, 2018; Lo \& Wang, 2004; Mandell et al., 2018; Mental Health Commission of Canada, 2019; Ng, 2012; Picot \& Lu, 2017; Um \& Lightman, 2016). In a study on chronic low income among immigrants in Canada, researchers found that over half of the older immigrant population suffered from chronic low income, compared to just two per cent of their Canadian-born counterparts (Lo \& Wang, 2004; Picot \& $\mathrm{Lu}, 2017)$. Interestingly, Picot and $\mathrm{Lu}$ (2017) concluded that the rate of chronic low income varied depending on the study population's place of birth.

Income, education and employment are interrelated factors in determining an older immigrant's financial stability. With the transition into pre-retirement, full retirement or the inability to sustain full- 
time employment as an older recent immigrant, it is common to see a decrease in the average income in this subgroup. In the peak earning years, between 45 and 54, the average income in Ontario in 2017 was around $\$ 68,000$, but gradually declines as age progresses (Statistics Canada, 2019). Racialized poverty in older recent immigrants, especially visible minorities, adversely affects their settlement transition, and first-generation or recent older immigrants are the most susceptible to racialization. Chronic low income is more prevalent in racialized older recent immigrants than in non-racialized or long-standing older immigrant groups (Creese, 2007; Galabuzi, 2006; Mandell et al., 2018; Picot \& Lu, 2017).

\subsubsection{Devaluation of education and limited employment}

An individual's income generation is typically correlated to the level of education attained, and as such, has implications on immigrant populations at large. Immigrants with higher levels of education tend to avoid chronic low income, however, while there has been a recent rise in immigrant education achievement, the income of this cohort has declined (Preston et al., 2010; Picot et al., 2007). Pendakur and Pendakur (2011), Creese (2007) and Galabuzi (2006) contend that there are three factors which influence immigrant earnings: devaluing foreign labour, lack of knowledge of an official language, and fewer low-wage employment opportunities (as cited in Lo \& Wang, 2004).

Furthermore, scholars have consistently found that the labour market disvalues immigrants regardless of their education achievement, particularly recent older immigrants, even though they were found to be proportionately more educated than their long-standing counterparts ( $\mathrm{Ng}$, Lai, Rudner \& Orpana, 2012; Preston et al., 2010). There is however evidence that suggests that economic prosperity becomes optimal for those older immigrants that are long-standing (Mandell et al., 2018). Additionally, Picot and Lu (2017) found that the income gap between recent and long-standing immigrants in the low income class was marginal, and proportionately higher than their Canadian-born counterparts, suggesting that income inequality might be lasting and perpetuated by racialized poverty (Lo \& Wang, 2004).

\subsubsection{Ethnocultural and linguistic diversity amongst older immigrants}

Recent older immigrants, who land and settle as an individual aged 65 and above, encounter the same set of settlement barriers as their long-standing counterparts did, but at a higher degree of oppression. Learning outcomes are predicated by cognitive capabilities which are influenced by age (Edmonston, 2016). For those immigrants that have not navigated through successive stages of learning opportunities, like the Canadian education system, employment, income and language proficiency prospects diminish (Edmonston, 2016). Thus resulting in more implications and negative impacts on their adjustment and integration into Canadian society. Challenges such as economic insecurity, devaluation of education, social isolation, and inadequate governmental supports are exacerbated by linguistic and cultural 
differences. Recent older immigrants are not as fluent in official languages as their long-standing counterparts, which results in a reliance on intergenerational support to navigate social services, public transportation and the labour market, which inherently lessens their independence and perceived value, thus depressing income (Dempsey, 2005; Lo \& Wang, 2004; Mandell et al., 2018). Mandell et al. (2018) found that a lack of knowledge of English or French is particularly prevalent in recent older immigrants, as their mother-tongue was likely to have been exclusively used in their birth country. Comparatively, long-standing older immigrants who landed more than 20 years ago are more likely to have developed fluency at some capacity since settlement (Chui et al., 2006; Creese, 2010; Gou, 2013; Government of Canada, 2005). Moreover, recent older adults are inadequately serviced by settlement supports as a result of settling in newly developed communities where insufficient knowledge of how and where to access services that are culturally and linguistically appropriate is impeding (Mandell et al., 2018).

When examining Chinese immigrants in Canada and Toronto, a large body of literature (Chow, 2010; Lai, 2000a; Lai, 2000b; Lai, 2003; Lai \& Yuen, 2003; Sadavoy, Meier \& Ong, 2004; Taylor, Taylor-Henley \& Doan, 2005) is present, hence, the high proportion of immigrant populations originating from an Asian country of origin. These studies find that the devaluation of language and culture is the main driver behind degraded mental health and well-being of older immigrants. Additionally, the immigrants that originate from China are not a homogenous group of individuals and have diverse regional origins, ranging from mainland Chinese, to Hong Kong to Taiwan, whose languages, traditions and cultures are all different (Lo \& Wang, 2004). Grouping these individuals under the umbrella of Chinese fails to recognize their in-group variations (Lo \& Wang, 2004). In relation to the experiences of older Chinese immigrants in Toronto and Canada on mental health and service issues Guruge, Thomson and Seifi (2015) found that the devaluation of language and culture is the main driver behind degraded mental health and well-being of older immigrants. Moreover, it is difficult to apply the influence that culture could have on mental health due to the ethnocultural diversity present in Canadian populations.

The 1991-1995 Immigration Plan produced by Canada's federal government included the Language Instruction for Newcomers to Canada program, which was initiated in 1992. The objectives of this program appears to provide educational opportunities for recent immigrants to assimilate into Canadian society by learning basic communication skills in English and French, understanding Canadian law structures, and understanding how to navigate basic social interactions, like shopping, banking or taking public transit (Guo, 2013; Mandell et al., 2018). Unfortunately, these services were not ethnoculturally relevant and promoted values that were derived from the white, middle-class experience, which is problematic and could result in a decline in participation (Guo, 2013; Laher, 2017; Mandell et al., 2018). Moreover, opportunities to learn and practice basic communication in one of Canada's official languages did not result in a decline of discrimination against immigrant labour, as studies indicate that 
English and French speaking immigrants who retained an accent were still ostracized and faced challenges navigating the labour and real estate markets (Creese, 2010).

\subsubsection{Accessible information and services}

Language barriers, multigenerational living, and sociocultural differences compound a recent older immigrant's ability to access not only relative, but accurate information on settlement programming and (City of Toronto 2017a; Mandell et al., 2018; McDonald et al., 2001; Stewart et al., 2011). Hence, program administrators, community leaders and regional governments should better understand the ethnocultural and linguistic composition of their populations to facilitate culturally appropriate services. Additionally, the channels in which an older immigrant is comfortable accessing information must be thoroughly considered. As suggested by Gallagher et al., (2006), older adults are anxious when expected to use technology to source information, as their unfamiliarity and inaccessibility to computers and internet act as technological barriers (as cited in Mandell et al., 2018). Informative print materials should be made more readily available and circulated to specific recent immigrant communities. An understanding of their spatial distribution is critical for social, cultural, and governmental services to evaluate targeted marketing strategies.

\subsection{Spatial distribution of immigrants in Toronto CMA}

Scholars who have researched the settlement patterns and social integration of immigrants in urban settings found relationships between period of immigration and spatial concentration (Hiebert, 2015; McDonald, 2004; Murdie, 2008; Simich, 2000; Vezina \& Houle, 2017). In general, the literature suggests that the vast majority of immigrants arriving in Canada settling in a CMA (specifically, Toronto, Montreal or Vancouver) and are rarely discovered to have settled outside of a CMA (King, 2009). Recent and established immigrants were typically found to not necessarily settle in clusters within the urban centres, but were distributed across the CMA in regions on the peripheral of the urban boundaries (Simich, 2000; Vezine \& Houle, 2017).

Vezina and Houle's (2017) study on settlement in three of Canada's largest CMAs suggest that suburban areas or areas outside of the central municipality are more appealing to all immigrant groups, in particular, long-standing immigrant settlement between 2001 and 2011. In Markham, Richmond Hill, Mississauga and Brampton, the population composition of each peripheral municipality was greater than $50 \%$ immigrant $(57.9 \%, 54.9 \%, 52.9 \%$ and $50.6 \%$ respectively), which surpassed the central municipality of Toronto's immigrant composition of $48.6 \%$ (Vezina \& Houle, 2017). Older immigrants that live in suburban regions, typically cluster in ethnocultural enclaves, while smaller portions of older immigrants in rural regions are scattered (Hiebert, 2015; Murdie, 2008; Vezina \& Houle, 2017). Throughout all of the 
relevant research examined, there was a lack of examination into the ethnocultural-specific makeup of the older immigrant adult population and where they landed spatially once they immigrated to the CMAs. As such, the distribution and concentration of residential uptake is largely influenced by the intersecting factors of ethnocultural origins, period of immigration and socioeconomic status.

\subsection{Summary of literature review}

The challenges encountered by immigrants when they migrate to Canada are compounded by age, ethnocultural origin, racialization, socioeconomic status and period of immigration. Recent older immigrants seek to be better understood in their communities by their fellow residences, local and regional governments and the Canadian society at large, while long-standing older immigrants have endured the challenges with settlement but throughout their tenure in Canada, have developed language skills to give them a competitive advantage (Zou \& Fang, 2017). Foreign-born older adults are faced with language barriers, cultural deterioration, chronic low income, and devaluation of education, which perpetuates their isolation and reluctance to integrate into Canadian society. Most of these factors are compounded by low income, and by large, the three most influential factors which affect earnings are devaluing foreign labour, lack of knowledge of an official language, and fewer low-wage employment opportunities (Pendakur \& Pendakur, 2011; Creese, 2007; and Galabuzi, 2006 as cited in Lo \& Wang, 2004).

Examining the socioeconomic and ethnocultural characteristics of the older immigrant population and acknowledging the differences in experience encountered by recent and long-standing immigrants and where they reside is a critical topic of research missing in this scholarship. Moreover, institutional, systemic and geographic barriers, like government funded programs, racialization and the spatial clustering of ethnocultural enclaves further exacerbate the challenges associated with settlement. Overall, scholarship suggests that older immigrants, particularly, those that have immigrated most recently, are disproportionately more susceptible and impacted by the barriers they encounter once they touch Canadian soil. Hence, there is a need for understanding their spatial concentration within the Toronto CMA and in relation to their long-standing counterparts. 


\section{Data}

\subsection{Study area and study population}

The Toronto Census Metropolitan Area (CMA) is the most populated CMA in Canada with a 2016 population of $5,928,040$. According to Statistics Canada, it was the most attractive immigrant destination in Canada in 2016 (Statistics Canada, 2018a). This study area covers $5,905 \mathrm{~km}^{2}$ in Southern Ontario and is banked to the south by Lake Ontario. It consists of 14 peripheral municipalities that surround the central municipality of Toronto, with older immigrant population concentrations located within the urban centres and concentration decreases towards the suburban and rural regions (Figure 1) (Statistics Canada, 2016). The Toronto CMA population is largely characterized by its diversity, with $51.3 \%$ of the population self-

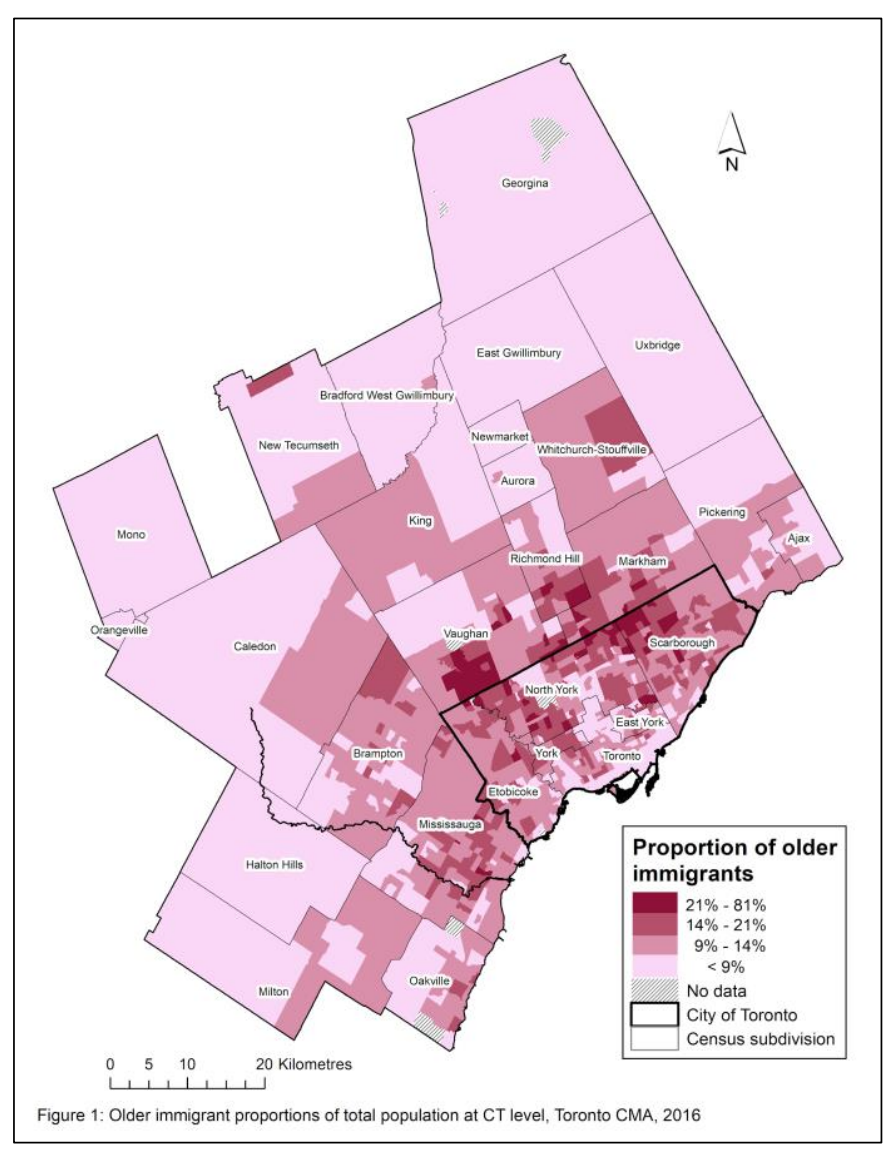
reporting as visible minorities, of which 71.5\% have ethnocultural origins from Asia (South Asian, Chinese, Filipino, Southeast Asian, West Asian, Korean, and Japanese) and $46.1 \%$ of the population are immigrants. Increased levels of immigration are being observed across Canada, where the immigrant population grew from $19.8 \%$ in 2006, to $20.6 \%$ in 2011, to $21.4 \%$ in 2016 (Statistics Canada, 2013). In Toronto, the immigrant population grew from $45.7 \%$ in 2006 , to $46 \%$ in 2011 , to $46.3 \%$ in 2016 , which is a remarkably high proportion of the total population being foreign-born (Statistics Canada, 2016; Statistics Canada, 2018b). In terms of socioeconomic characteristics, Toronto has a higher rate of low income (20.2\%) relative to Canada (14.2\%), according to the 2016 census (City of Toronto, 2017b). The distribution of income and the concentration and clustering of ethnocultural subgroups suggest that the Toronto CMA is an ideal study area for this analysis.

The study populations examined in this research paper include older immigrants, older nonimmigrants and older immigrants from four specific immigrant communities - Chinese, Italian, Portuguese and South Asian. Specifically, it includes foreign-born individuals over the age of 65 and older non-immigrant adults, who are Canadian-born individuals over the age of 65 in the Toronto CMA. 
Older immigrants from the four largest ethnocultural immigrant groups in the Toronto CMA (Chinese, Italian, Portuguese and South Asian) are also evaluated. These four ethnocultural subgroups are selected as they represent almost $50 \%$ of the ethnocultural diversity for older immigrants in the Toronto CMA. The four ethnocultural groups are further subgrouped into recent and long-standing immigrant groups, based on the period of immigration. As there was a shift in sending-countries away from European nations and towards Asian nations, the long-standing and recent older immigrant subgroups each have two ethnocultural demographics (Statistics Canada, 2013). The long-standing older immigrants' subgroups, who arrived before 2000, are comprised of the Italian and Portuguese older immigrant groups. The recent older immigrants, arriving after 2001, is comprised of Chinese and South Asian older immigrants. Recent criticism in the perception that South Asians or Chinese are each a homogenous cohort must be acknowledged, as this research paper uses these two overarching ethnocultural classes to group what, in reality, are cohorts with high in-group variation (Lo \&Wang, 2004; Mathews, 2000). Due to the exploratory nature of this research paper, and the methodological limitations associated with using small sized study populations, these two subgroups are used.

\subsection{Demographic Data}

Data sources used in this analysis are detailed in Table 1 below. The primary data source used is a custom cross-tabulation in Beyond 20/20, obtained from Statistics Canada. This dataset is based on the 20\% sample of the population aged 15 years and above in private households for the Toronto CMA, and is provided by Dr. Lu Wang. Derived from the 2016 Census, it provides the geography of seven dimensions of demographic characteristics in the preceding variables at the census tract (CT) level: ethnic origin, sex, age groups, immigrant status and period of immigration, total income groups, language spoken most often at home, and highest certificate, diploma or degree.

Table 1: Data and data sources

\begin{tabular}{|l|l|l|l|}
\hline Data Source & Year & Type & Description \\
\hline Boundaries & 2016 & $\begin{array}{l}\text { Vector polygon } \\
\text { shapefiles }\end{array}$ & $\begin{array}{l}\text { CT, CMA and City of Toronto level } \\
\text { boundary files }\end{array}$ \\
\hline Statistics Canada & \multicolumn{3}{|l|}{} \\
\hline Demographic data & 2016 & Census tract level, IVT & $\begin{array}{l}\text { Socioeconomic and ethnocultural } \\
\text { variables in crosstab form }\end{array}$ \\
\hline $\begin{array}{l}\text { Custom Statistics } \\
\text { Canada }\end{array}$ & 2016 & Census tract level, XLS & Material deprivation index \\
\hline Marginalization Index & &
\end{tabular}


A full list of variables used can be seen in Table 2 below, which includes custom variables that were created by aggregating or disaggregating variables within similar themes.

Table 2: Variables used in the study

\begin{tabular}{|c|c|}
\hline Dimension and variable & Derived or provided variable \\
\hline \multicolumn{2}{|l|}{ Age group } \\
\hline $65+$ & Derived: $(65-64+>75)$ \\
\hline $65-74$ & Provided \\
\hline $75+$ & Provided \\
\hline \multicolumn{2}{|l|}{ Gender } \\
\hline Female & Provided \\
\hline Male & Provided \\
\hline \multicolumn{2}{|l|}{ Income variables } \\
\hline LICO-AT & Derived: a weighted average was applied \\
\hline Low income $(<\$ 19,999)$ & Derived: $(<\$ 10,000+\$ 10,000-\$ 19,999)$ \\
\hline $\begin{array}{l}\text { Medium income }(\$ 20,000- \\
\$ 49,999)\end{array}$ & $\begin{array}{l}\text { Derived: }(\$ 20,000-\$ 29,999+\$ 30,000-\$ 39,999+\$ 40,000- \\
\$ 49,999)\end{array}$ \\
\hline High income $(\$ 50,000+)$ & $\begin{array}{l}\text { Derived: }(\$ 50,000-\$ 59,999+\$ 60,000-\$ 69,999+\$ 70,000- \\
\$ 79,999+\$ 80,000-\$ 89,999+\$ 90,000-\$ 99,999+>\$ 100,000)\end{array}$ \\
\hline \multicolumn{2}{|l|}{ Education attainment } \\
\hline $\begin{array}{l}\text { No certificate, diploma or } \\
\text { degree }\end{array}$ & Provided \\
\hline Secondary (high school) & Provided \\
\hline Post-secondary & Provided \\
\hline \multicolumn{2}{|l|}{ Ethnocultural origin } \\
\hline Chinese & Provided \\
\hline Italian & Provided \\
\hline Portuguese & Provided \\
\hline South Asian & Provided \\
\hline \multicolumn{2}{|l|}{ Language most spoken at home } \\
\hline Official language & Derived: (English + French) \\
\hline English & Provided \\
\hline French & Provided \\
\hline Non-official language & Provided \\
\hline $\begin{array}{l}\text { English and non-official } \\
\text { language }\end{array}$ & Provided \\
\hline \multicolumn{2}{|l|}{ Period of immigration variables } \\
\hline Long-standing immigrant & Derived: $(<1981+1981-1990+1991-2000)$ \\
\hline Before 1981 & Provided \\
\hline $1981-1990$ & Provided \\
\hline 1991-2000 & Provided \\
\hline Recent immigrant & Derived: $(2001-2010+2011-2016)$ \\
\hline $2001-2010$ & Provided \\
\hline $2011-2016$ & Provided \\
\hline
\end{tabular}


Four ethnoculturally-specific study populations are examined in this study and consist of older immigrants with ethnocultural origins of Chinese, Italian, Portuguese and South Asian. These subgroups were selected as they represent the countries that hold the highest share of the immigrant populations in the Toronto CMA, as shown in Figure 2. They are representative of established immigrants (Italian and Portuguese) and recent immigrants (Chinese and South Asian). Additionally, older non-immigrants are a secondary study group used when comparing socioeconomic, ethnocultural, and spatial characteristics of older immigrants. These study populations are extracted from the custom cross-tabulation data using multiple layers of disaggregation, such as immigrant status, ethnocultural origin, and age group.

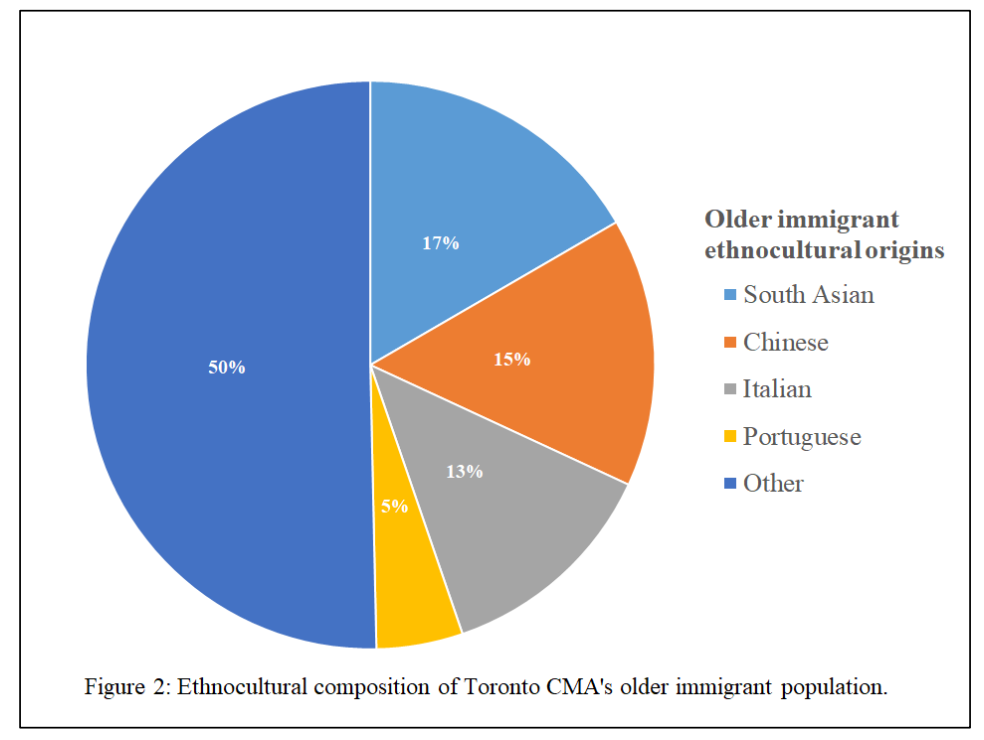

Assessing the use of age cutoffs in other literature suggests that the widely accepted age when analyzing older adult populations is 65 years old and above, thus older adults in this analysis are individuals aged 65 and above. However, this analysis incorporates immigrant populations from four ethnocultural backgrounds who might have migrated from developing regions of the world and a broader definition of older adults was considered. Research out of the World Health Organization (2002) on age classification structures suggested using the age cutoff of 50 years old when analyzing older adult populations to account for the varying life expectancies and cultural differences throughout developing nations. Other literature uses multiple life-stage groups (55-64, 65-74, 75-84 and 85+) to explore in-group differences based on age groups for individuals in the 65 and above category (Sekhar, Shipton \& Bruce, 2010). While multiple age subgroups would determine in-group differences based on age, given the reasons above the age group selected for this analysis is individuals that are 65 years or older, which is referred to as older adults.

Age data was provided in six age groups (as outlined in Table 2) including a 65-74 age group and a 75 years and over age group. Both groups are aggregated to create the 65 and above age group which is 
the primary constraint applied to all data processing, as this research is seeking to examine various subgroups of older adults.

As defined by Statistics Canada, an immigrant is a foreign-born individual that has migrated to Canada (Statistics Canada, 2018). Immigrant status was the secondary constraint applied to all data processing, as this research is seeking to examine various immigrant subpopulations. Additional immigrant-related data was provided, including period of immigration. Although there were five ten-year long landing period attributes, they are aggregated into the recent immigrant groups (those landing before 2000) and the long-standing immigrant groups (those landing in 2001 or after).

Ethnocultural origin data provided includes individuals with origins of Chinese, Italian, Portuguese and South Asian. While nine ethnocultural groups were provided in the full data set, these four groups were selected because they represent the largest share of immigrants, that are traditionally seen as long-standing (Italian and Portuguese) and recent (Chinese and South Asian) immigrant groups. Of the select subgroups, 99\% and 98\% of Italian and Portuguese older immigrants, arrived before 2000, and $20 \%$ and $26 \%$ of the Chinese and South Asian older immigrants, arrived after 2001 (Statistics Canada, 2018d).

Education, income and marginalization attributes are used in this research paper to illustrate socioeconomic status and vulnerability. Income data is provided in intervals of $\$ 10,000$ ranging from $<\$ 10,000$ to $\$ 100,000+$. Three income classes are created with these income intervals: low income, medium income and high income. While there is limited scholarship on income class aggregation for older immigrants, Statistics Canada and the Canadian government published the use of different income class cutoffs which are employed in this research. The Ontario Ministry of Health and Ministry of LongTerm Care used $\$ 19,300$ as the indicator for low income in welfare distribution, thus, the low income range in this research is $<\$ 19,999$. Picot and Hou (2014) found that the average income of an individual aged 65 years in 2017 is $\$ 42,500.00$, thus, the medium income class range is $\$ 20,000$ to $\$ 49,999$. Additionally, they found that the median income of an older adult is $\$ 26,100$. Both the average and median income values determined by Picot and Hou (2014) fall within the medium income class used in this research paper. Picot and Hou (2014) also indicated that their high income class cutoff was twice the median value, which would be $\$ 52,200$, therefore the high income class in this research paper is $\$ 50,000$ and above.

In addition to the three new income classes, the dataset includes values for the low income cut-off after tax (LICO-AT), which is an income inequality measure that indicates economic vulnerability or deprivation (Statistics Canada, 2012b). According to Statistics Canada (2012b), the LICO- AT value is a percentage of the population that uses more of its income on basic amenities (like food, shelter and clothing) compared to an average family. If a LICO-AT percent of a subgroup is $15 \%$, for example, then 
it suggests that $15 \%$ of the subgroup's total population are economically disadvantaged. These values are derived from Family Expenditure Surveys and Statistics Canada census income data and the Survey of Consumer Finances (Statistics Canada, 2012b). For scholarly purposes, it is the most established poverty measure in Canadian poverty research, and is used as a measure of poverty by the Central Intelligence Agency (CIA) in the United States of America (Albridge, 2017). This variable is particularly significant as older adult poverty has social and fiscal implications, and will continue to be problematized as higher proportions of populations continue to age rapidly (World Health Organization, 2014). Since LICO-AT values are already in percent form, the $65-74$ and $75+$ need to be summed, then a weighted average is applied.

Education variables included in this analysis account for a wide range of education attainment. The three education variables selected are: no certificate, diploma or degree, which represents less than a high school education; secondary certificate of equivalent, which represents a high school education; and post-secondary certificate, diploma or degree.

Considering the provision of data in a cross-tabulation file, various attributes can be layered in Beyond 2020 to extract data on a particular subgroup for further examination. For example, to determine values for the long-standing Chinese older immigrant population, four layers were imported into the Beyond 2020 content window: count of immigrants, count of Chinese, count of 65-74 age, count of 75+ age group, count of landing period before 1981, count of landing period from 1981-1990, and count of landing period from 1991-2000. The resulting cross-tabulation is exported for further processing in Microsoft Excel. This method is applied throughout the data processing stage.

The Ontario Marginalization Index is a data set derived from Statistics Canada's 2016 census. It was created by Ontario Public Health to explore level of marginalization and inequalities across area units in Ontario. Only the material deprivation dimension is used to determine if there is any spatial overlap between material deprivation and older immigrant clusters (Matheson, 2016). This dimension is generated from a combination of income, housing, education, and family structure measures and is an indicator of an individual's access to material amenities (Matheson, 2016).

All variables included in this are at the census tract level, which typically consist of between 2,500 and 8,000 individuals per tract (Statistics Canada, 2018c). Although it is not the finest geographic unit, using a smaller resolution would produce a higher number of zeros in the data set which is not a suitable option for this study.

\section{Methods}

This analysis represents a pilot study to fill in the gap found in the already limited scholarship on the characterization, spatial distribution and in-group differences of older immigrants in the Toronto CMA. It 
attempts to understand the four subgroups based on the notion of spatial heterogeneity as a phenomenon influenced by culture and time. The unit of geography that is used in this analysis is the Census Tract (CT). There are $1151 \mathrm{CTs}$ in the Toronto CMA, which range in population from 265 to 17,875 and is used to address the research questions.

\subsection{Cross-tabulations and line graphs based on custom census}

Describing population compositions for a well-defined cohort of individuals are traditionally presented in text, tabular or chart-based forms, particularly when data summaries are involved (Ng, Lai, Rudner \& Orpana, 2012; Dempsey 2005; Frey, 2000; Lo \& Wang, 2004). When describing large, perhaps complicated data sets, text might not be the most effective medium to adequately convey a narrative. In these instances, accompanying tables and charts are quite effective and can help to reveal patterns in the data at-a-glance. Recently, pie and stacked bar charts have been employed to illustrate the distribution of older adults by immigrant status and period of immigration based on the Canadian CMA in which they migrated to (Ng, Lai, Rudner \& Orpana, 2012). Differentiating the economic characteristics within ethnocultural subgroups, Lo \& Wang (2004) used multivariable line charts to illustrate numerous dimensions of analyses, allowing for the exploration of cross tabular data in a visual manner. This research paper adopts a similar approach to present the findings on older immigrant population characteristics in the Toronto CMA. These charts will use line colour and marker shape to represent several variables being visualized.

\subsection{Spatial Methods}

Spatial distributions of older populations have commonly been measured independently using population ratios, proportions, hot spot mapping and location quotients. However, using a particular measure in isolation might limit the breadth of findings, particularly any insights into the urban and rural divide that is present in many spatial demographic analyses (Chiode, Morita, Shiode \& Okunuki, 2013). Using a variety of these methods in tandem can help corroborate findings and produce a more holistic and comprehensive assessment of the spatial patterns being observed. This research paper examines the six study populations using proportion mapping, Getis-Ord Gi* hot spot analysis and location quotients.

To explore and compare the spatial distribution of Toronto CMA's older immigrant and nonimmigrant populations, choropleth maps are produced in ArcMap. This method is also applied to the LICO-AT distribution to explore the spatiality of older immigrant and older non-immigrant poverty. Additionally, to understand the overrepresentation and underrepresentation of the four ethnocultural older immigrant groups, a Location Quotient (LQ) model is applied. LQ is a measurement used in economic and demographic analytics to measure the relative and unique concentration of a particular population 
group in small geographic regions relative to their concentration in the overall study area. This method has been used in various fields including demographic analysis (Asthana, Gibson, Moon, Brigham, \& Dicker, 2004), crime analytics (Andresen, 2007), immigrant settlement patterns (Biase \& Harald, 2005), and older population distributions (Seguin, Apparicio, Riva \& Negron-Poblete, 2016; Lambert, Wilcox, Clark, Murphy \& Park, 2010). The LQ identifies the proportion of the older immigrant study group population in a particular CT divided by the total observed value for the Toronto CMA and is represented by the equation, $\mathrm{LQ}_{\mathrm{i}}=\left(x_{i} / t_{\mathrm{i}}\right) /(X / T)$, where:

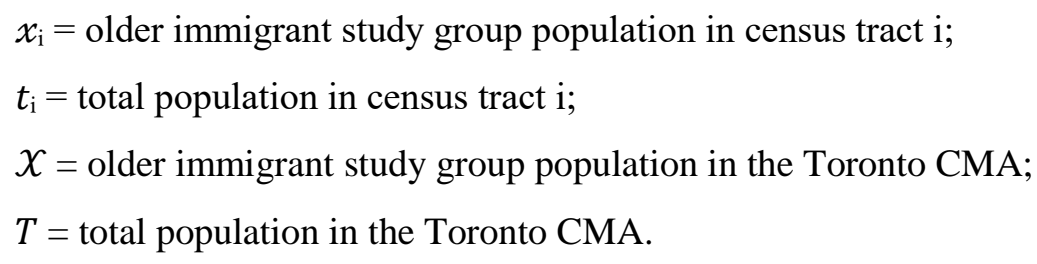

LQs assigns a decimal numeral to each CT, with the lowest value of 0 representing no presence of the particular study group relative to the larger study area, and the highest value representing an extreme over representation of the study population in the CT, relative to the CMA's composition. LQs between 0 and 0.8 denote an underrepresented study population, and those between 0.8 and 1 slightly represented, while LQs between 1 and 1.2 represent moderate representation and values above 1.2 denote an overrepresentation. However, most studies use two classes, which indicate underrepresentation $\left(\mathrm{LQ}_{\mathrm{i}}<1\right)$ or overrepresentation $\left(\mathrm{LQ}_{\mathrm{i}}>1\right)$ (Cromley \& Hanink, 2012). Since the distribution of the LQs have large ranges, four classes are used in this research paper.

In some instances, LQs that are in the double-digits would indicate a geographic unit that is extremely over representative of a particular study group and in this study the value of 5.2 is used to denote extremely over represented. All four subgroups have values over 5.2 and it allowed for proper visualization of the extremely overrepresented. For example, if an LQ for a CT is 3.5, it means that the study group is 3.5 times more represented in that particular CT than they are in the entire Toronto CMA. Location quotients are generated on the Chinese, Italian, Portuguese and South Asian older immigrant populations and mapped to identify the representation per CT relative to the entire CMA. Both the choropleth and location quotient methods are not statistical in nature, hence, a spatial statistic is further employed to corroborate the patterns observed in the aforementioned approaches.

The Getis Ord Gi* method is a spatial statistic developed in the early 1990s that is a commonly used technique employed by scholars to investigate the location of spatially concentrated or intense population clusters (Anselin, 1995; Mazzulla \& Forciniti 2012; Malczewsi, 2010; Getis \& Ord, 1992; Kang, Cho \& Son, 2018; Somenahalli, Shipton \& Bruce, 2010). A 3,300m fixed distance band parameter 
is applied as it was used in similar research designs, on similar sized geographic units (Varga, Pearl, McEwen, Sargeant, Pollari, \& Guerin, 2015; Yamashita \& Kunkel, 2012). ArcMap's Hot Spot Analysis Getis-Ord Gi* tool is applied to ON-Marg's material deprivation index, and the recent and long-standing older immigrant populations for the Chinese, Italian, Portuguese and South Asian subgroups. This is used to support findings from choropleth and location quotient mapping by allowing for the objective analysis of statistically significant clusters. As this research paper seeks to identify the concentrations of highly clustered immigrant subgroups, only the hot spots will be visualized, which represent the $99 \%, 95 \%$ and 90\% confidence levels. Cold spots will therefore not be used in this analysis. Regarding methodological limitations, the recent older immigrant subgroup hot spot maps should be reviewed with caution as their study population was significantly smaller than the sample size of the long-standing subgroups.

\section{Results}

The preceding section outlines the results of the population and spatial analysis of the various ethnocultural subgroups which are discussed based sequence of analysis. The socioeconomic and ethnocultural population characteristics of the older non-immigrant and immigrant populations and their spatial patterns are discussed first followed by a discussion on the socioeconomic and ethnocultural compositions and spatial distribution of the four ethnocultural subgroups (Chinese, Italian, Portuguese and South Asian), according to their recent and long-standing settlement categories.

\subsection{Comparing older immigrants and older non-immigrant}

\subsubsection{Variation in socioeconomic and demographic composition}

As shown in Table 3, the demographic characteristics of the older non-immigrants and older immigrants suggest that they are vastly different in composition. The vast majority of older individuals in the Toronto CMA are immigrants (71\%) while just over one-fourth (28\%) are Canadian-born. Evaluating population characteristics is a valuable method to understand the composition of a population, which can perhaps provide some context to the spatial patterns that these study populations exhibit. In terms of age and gender composition, both older non-immigrants and older immigrants exhibited similar proportions of individuals in the 65-74 age group and the 75 and above age groups. Additionally, the gender proportions indicate that there is a small majority of females in both the older immigrant and older non-immigrant populations (55\% and 55\%, respectively). 
Table 3: Socioeconomic and demographic dimensions of older immigrants and non-immigrants.

\begin{tabular}{|c|c|c|c|c|c|c|}
\hline \multirow{2}{*}{ Dimension } & \multicolumn{3}{|c|}{ Older non-immigrants } & \multicolumn{3}{|c|}{ Older immigrants } \\
\hline & \# & $\begin{array}{c}\% \text { older } \\
\text { pop }\end{array}$ & $\begin{array}{c}\% \\
\text { subgroup }\end{array}$ & \# & $\begin{array}{c}\% \text { older } \\
\text { pop }\end{array}$ & $\begin{array}{c}\% \\
\text { subgroup }\end{array}$ \\
\hline \multicolumn{7}{|l|}{ Age } \\
\hline $65+$ population & 230,475 & $28 \%$ & $100 \%$ & 581,540 & $71 \%$ & $100 \%$ \\
\hline $65-74$ years old & 136,100 & $17 \%$ & $59 \%$ & 334,400 & $41 \%$ & $58 \%$ \\
\hline $75+$ & 94,375 & $12 \%$ & $41 \%$ & 247,140 & $30 \%$ & $43 \%$ \\
\hline \multicolumn{7}{|l|}{ Gender } \\
\hline Male & 103,040 & $13 \%$ & $45 \%$ & 264,590 & $32 \%$ & $46 \%$ \\
\hline Female & 127,425 & $16 \%$ & $55 \%$ & 316,945 & $39 \%$ & $55 \%$ \\
\hline \multicolumn{7}{|l|}{ Income } \\
\hline Low & 40,780 & $5 \%$ & $17.7 \%$ & 377,835 & $33 \%$ & $45.6 \%$ \\
\hline Medium & 96,845 & $12 \%$ & $42.0 \%$ & 130,535 & $26 \%$ & $37.0 \%$ \\
\hline High & 92,830 & $11 \%$ & $40.3 \%$ & 43,135 & $12 \%$ & $17.4 \%$ \\
\hline \multicolumn{7}{|l|}{ Education attainment } \\
\hline No education & 44,655 & $6 \%$ & $19 \%$ & 197,620 & $24 \%$ & $34 \%$ \\
\hline Secondary education & 67,060 & $8 \%$ & $29 \%$ & 135,455 & $17 \%$ & $23 \%$ \\
\hline Post-secondary education & 118,760 & $15 \%$ & $52 \%$ & 248,465 & $31 \%$ & $43 \%$ \\
\hline \multicolumn{7}{|l|}{ Ethnocultural origin } \\
\hline South Asian & 170 & $0 \%$ & $0 \%$ & 96,645 & $12 \%$ & $17 \%$ \\
\hline Chinese & 950 & $0 \%$ & $0 \%$ & 88,965 & $11 \%$ & $15 \%$ \\
\hline Italian & 7,365 & $1 \%$ & $3 \%$ & 74,635 & $9 \%$ & $13 \%$ \\
\hline Portuguese & 170 & $0 \%$ & $0 \%$ & 28,535 & $4 \%$ & $5 \%$ \\
\hline \multicolumn{7}{|l|}{ Language spoken most at home } \\
\hline Official & 228,075 & $28 \%$ & $99 \%$ & 236,785 & $29 \%$ & $41 \%$ \\
\hline Non-official language & 945 & $0 \%$ & $0 \%$ & 293,290 & $36 \%$ & $50 \%$ \\
\hline English and non-official language & 970 & $0 \%$ & $0 \%$ & 50,290 & $6 \%$ & $9 \%$ \\
\hline
\end{tabular}


Regarding income distributions, overall, older immigrants are observed to be proportionately less wealthy than their Canadian-born counterparts. Of older non-immigrants, $17.7 \%$ fall in the low income class $(<\$ 19,999)$, compared to $45.6 \%$ of older immigrants. Older non-immigrants hold a proportionately higher share of the medium income class, as $42 \%$ of them fall in the medium income class $(\$ 20,000$ $\$ 49,999)$, compared to $37 \%$ of older immigrants. Lastly, $40.3 \%$ of older non-immigrants fall in the high income class $(\$ 50,000+)$, in sharp contrast to $17.4 \%$ of older immigrants. Isolating the highest income bracket, it was found that $11.5 \%$ of the older non-immigrant population earns more than $\$ 100,000$, while only $4.1 \%$ of older immigrants fall in this income class. The secondary income dimensions are the LICOAT prevalence values, which indicate that $10 \%$ of immigrants and $6 \%$ of older non-immigrants are below the low income cut off.

Another measure of socioeconomic status used in this study is education attainment, which follows a similar pattern as income, in that older non-immigrants appear to be more educated than their immigrant counterparts. Almost a fifth (19\%) of the older non-immigrants population have no formal education, compared to a third (34\%) of the older immigrant population. Non-immigrants also see a slightly higher proportion of their population educated at the high school or equivalent level (29\%) compared to a slightly lower proportion in the older immigrant population (23\%). Lastly, more than half $(52 \%)$ of the older non-immigrants population have a post-secondary certificate, diploma or degree, which includes a Bachelor's degree or a degree above Bachelor's, and similarly, the older immigrant population has a large proportion of post-secondary graduates (43\%).

How does education achievement influence income generation for immigrant and older nonimmigrants? As shown in Figure 3, the line plots suggest that although income increases relative to education, there are large income gaps between the older immigrant and older non-immigrant populations. This is most evident in the low income classes for all education levels, and also in the high income class for post-secondary education. Each education class is broken into three subsections, as indicated by the marker symbol. The circular marker represents low income $(<\$ 19,999)$, the square marker represents medium income $(\$ 20,000-\$ 49,999)$ and the diamond marker represents high income $(\$ 50,000+)$. The line colour indicates, in this instance, the older immigrant and older non-immigrant patterns. This symbology explanation applies to all multivariable line charts in this research paper. 


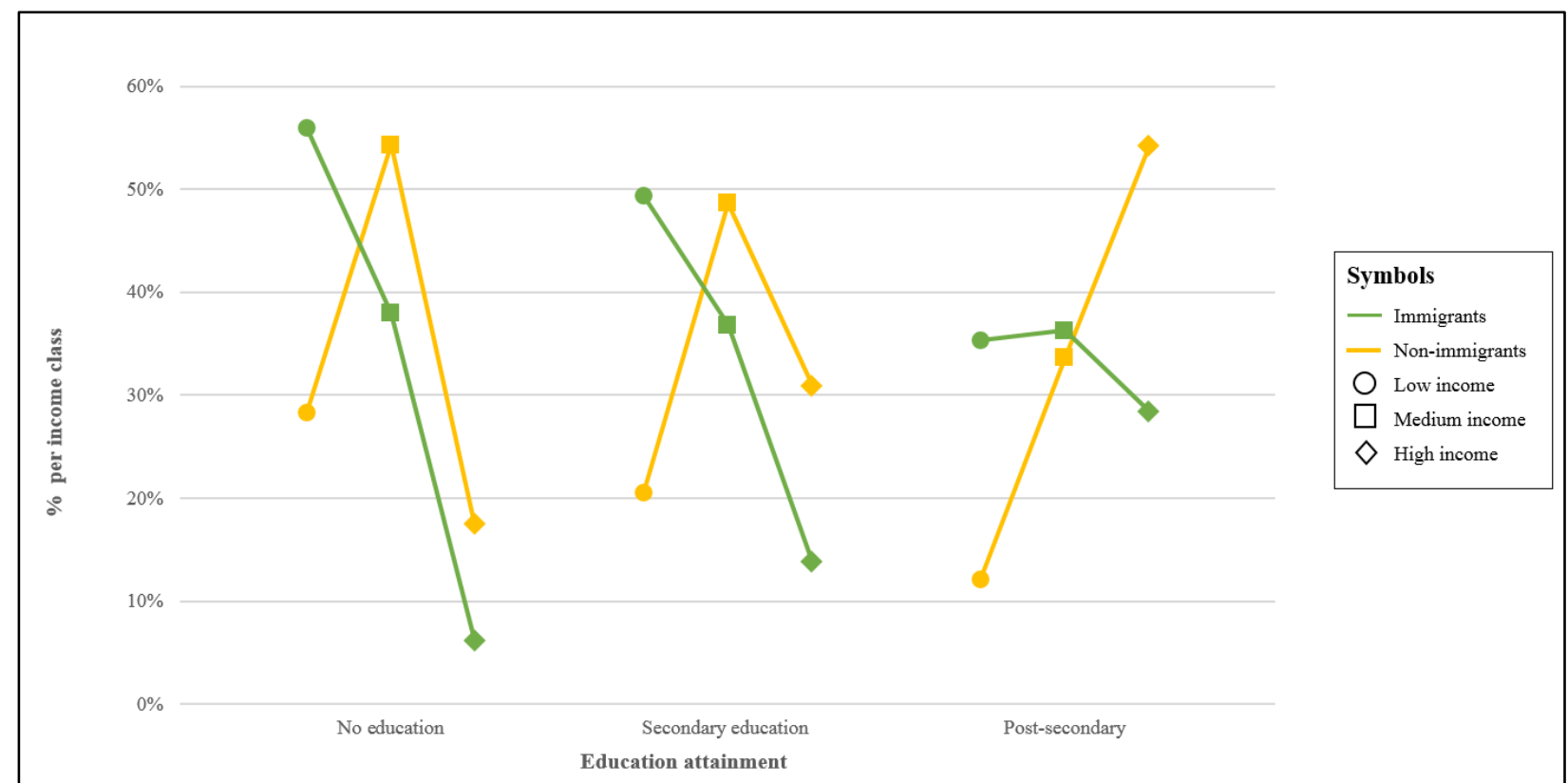

Figure 3: Comparing income of older immigrants and older non-immigrants based on education attainment.

Older immigrants with no education are proportionally more present in the low income class than their non-immigrant counterparts (55.9\% and $28.3 \%$, respectively). This is also present in the secondary education level, where $20.5 \%$ of older non-immigrant adults with a high school education earn less than $\$ 19,999$, compared to almost half $(49.4 \%)$ of the older immigrant population. Attaining a post-secondary degree appears to have the most impact on income for both groups. While 35.3\% of highly educated older immigrants are in the low income class, there are still $28.4 \%$ that fall in the high income class.

Comparably, highly educated older non-immigrants fair far better financially, as only $12.1 \%$ of them are low income earners, and more than half (54.2\%) are high income earners.

Ethnocultural characteristics of the older non-immigrant and immigrant population demonstrate in-group differences based on ethnocultural origin, language spoken and period of immigration. Table 3 presents the ethnocultural characteristics of these populations where findings suggest that the older nonimmigrant population is not ethnoculturally diverse, with the largest share assigned to Italian-Canadians (3\%). Additional proportions of the older immigrant population reveals that $17 \%$ are South Asian, $15 \%$ are Chinese, $13 \%$ are Italian and almost 5\% are Portuguese. The combination of older immigrants for these four ethnocultural subgroups represents just over half of the total older immigrant population in Toronto CMA.

Language spoken most often at home is also indicative of the variation across these two populations, where almost all of the older non-immigrants (99\%) speak an official Canadian language and less than $1 \%$ of this population speaks a non-official language. Conversely, less than half $(41 \%)$ of the 
older immigrant population speaks English or French, and 50\% speak mostly a non-official language at home.

\subsubsection{Variation in settlement patterns of older immigrants and older non-immigrants}

In terms of spatial patterns of the older immigrant and non-immigrant populations and their spatial overlap with areas of material deprivation can be seen in Figures $4 \mathrm{a}$ through 4e. Figure 4a presents the proportion of older non-immigrants per $\mathrm{CT}$ and $5 \mathrm{~b}$ presents the older immigrant hot spots. Older immigrants (Figure 4a) have proportionately high concentrations in both the City of Toronto and peripheral municipalities around its border. In Toronto, areas with high proportions are located adjacent to the downtown core, in areas like Scarborough in the east, North York in the north, and York in the west. Within these regions, the highest proportion of older immigrants appear to be concentrated in the neighbourhoods of West Hill, Malvern, Agincourt and Steeles, Parkdale, Eglinton West and Weston. Concentrations then appear to span northward, into Markham's neighbourhoods of Unionville and Greensborough, and through Vaughan's Maple, and Concord. Overall, there appears to be an arch-like pattern of older immigrant settlement around the downtown core, moving into the peripheral municipalities. This group is also largely absent in rural municipalities, like Uxbridge, Georgina, East Gwillimbury, New Tecumseth, Caledon, Mono, Halton Hills and Milton. These figures illustrate the proportion of older immigrants and areas in the CMA where there might be isolated enclaves of high proportions of older immigrants that are surrounded by CTs with low proportion of older immigrants. Statistically significant clustering of older immigrants is provided in Figure $4 \mathrm{~b}$, which yields similar spatial patterns, but emphasizes CTs with high older immigrant values surrounded by other CTs with high older immigrant values. The hot spots presented in Figure $5 \mathrm{~b}$ echo the general patterns found in Figure 4a. Approximately three distinct clusters of statistically significant hot spots of older immigrants are present. Beginning in the east, the largest cluster can be found starting in the inner suburbs located in the northern portions of Scarborough and North York, spanning north into most of Markham, Richmond Hill, and the southeastern section of Vaughan. The second largest cluster of high values is located on the west end of Toronto, encompassing northern portions of North York and Etobicoke's neighbourhoods of Weston, Rexdale, then moving north through southern Vaughan in areas like Woodbridge and Kleinburg. This cluster also spans into northeastern Brampton and one large CT in Caledon. The third cluster is located along the Credit River which transgresses through the Cooksville neighbourhood in Mississauga and in a small region of southwestern Brampton. 


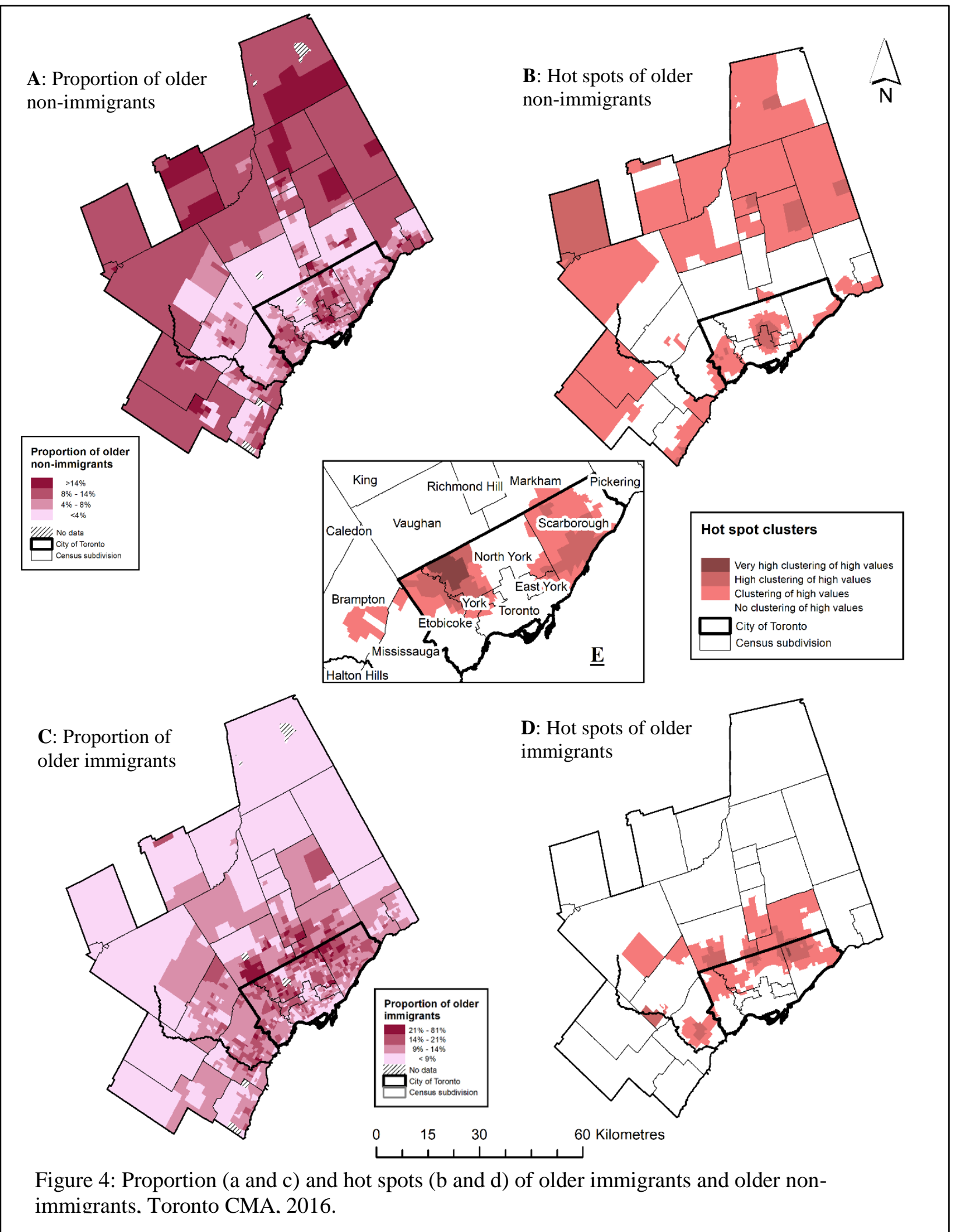

In stark contrast to the patterns and clusters observed in the older immigrant spatial distributions, Figure $4 \mathrm{c}$ and $4 \mathrm{~d}$ presents the proportions and clustering of older non-immigrants in the Toronto CMA. 
Figure $4 \mathrm{c}$ depicts the spatial distribution of older non-immigrants which exhibit high proportions in the downtown core, specifically along the Yonge corridor, with concentrations also observed along the Lakeshore, in Etobicoke and in general, most inner urban regions south of Highway 401. Additional concentrations are exhibited throughout most of the northern rural municipalities. In comparison to hot spot mapping, both mapping techniques yielded similar patterning. As shown in Figure 6d, the older nonimmigrant population have statistically significant clusters and are located in areas absent of older immigrant clusters. Linear clustering of high values along Toronto's lakeshore is observed, from Ajax in the east, through the Beaches neighbourhood in Toronto, then again found west of the Credit River in Mississauga, and continuing west along Oakville's lakeshore. A larger cluster is located in central Toronto, west of the Don Valley Parkway, beginning in the Yorkville and Annex neighbourhoods and running north through Summer Hill, Forest Hill, Rosedale and Lawrence Park. Another large cluster is located in the southern half of Etobicoke. Lastly, rural municipalities like Uxbridge, Georgina, East Gwillimbury, New Tecumseth, Caledon, Mono, Halton Hills and Milton observe statistically significant clustering of older non-immigrant adults.

Overall, when comparing the older immigrant hot spots (Figure 4b) with older non-immigrant hot spots (Figure 4d) a clear spatial delineation between the residential patterns of each subgroup is present. However, two small areas, one in the Port Union and Guildwood regions of southern Scarborough and the other in Humber Valley exhibit spatial overlap of both subgroups, suggesting that high clustering of these subgroups only co-reside in very few, and small regions of the CMA.

Examining spatial overlap of ON-Marg's material deprivation index with hot spots (Figure 4e) of older immigrant and non-immigrant groups suggests that older immigrant's residential hot spots overlap more with hot spots of material deprivation than that of older non-immigrants. Two large hot spots of material deprivation are located on each end of Toronto. The first is within Victoria Park Avenue on the west end, to Morningside Avenue on the east end, and the second is from just above Steeles Avenue in the north, down to the lake in the south, encompassing all of Scarborough. The northern segment of this hot spot overlaps with the southern segment of the largest older immigrant cluster. The second cluster of material deprivation is found in the north west end of the city, encompassing the Weston, Downsview, Jane and Finch, and Rexdale neighborhoods, and some of Malton, which also corresponds with clusters of older immigrants (as shown in Figure 4b). Conversely, the material deprivation hot spots do not appear to overlap with any older non-immigrant cluster, except for the cluster of older non-immigrants located along the lakeshore in the Guildwood and Port Union regions of eastern Scarborough. 


\subsubsection{Variation in settlement patterns of long-standing and recent older immigrants}

This section of the research paper will examine the settlement patterns of each ethnocultural subgroup based on those that are long-standing older immigrants or recent older immigrants. Figures $5 \mathrm{a}$ and $5 \mathrm{~b}$ present the settlement patterns of older immigrants that are long-standing (Figure 5a) compared to those that are recent (Figure 5b). Long-standing immigrants arrived in Canada before the year 2000, while recent older immigrants arrived between 2001-2016. It is evident that when comparing the two distributions below, both long-standing and recent older immigrants appear to have not settled in the same parts of the Toronto CMA. While there is a little overlap, the majority of the clusters are separate. Longstanding older immigrants (Figure 5a) appear to have clustered in inner urban regions north of Highway 401 into Vaughan, Richmond Hill and Markham. A second cluster is found in central Mississauga. Figure $6 \mathrm{~b}$ illustrates the settlement patterns of the recent older immigrant populations. This subgroup appears to be clustered predominantly in suburban regions outside of Toronto, encompassing the entirety of Markham and Richmond Hill, the western portion of Vaughan. A second cluster located in northern Mississauga and through most of Brampton, with the exception of the Bramalea neighbourhoods in the heart of Brampton. 


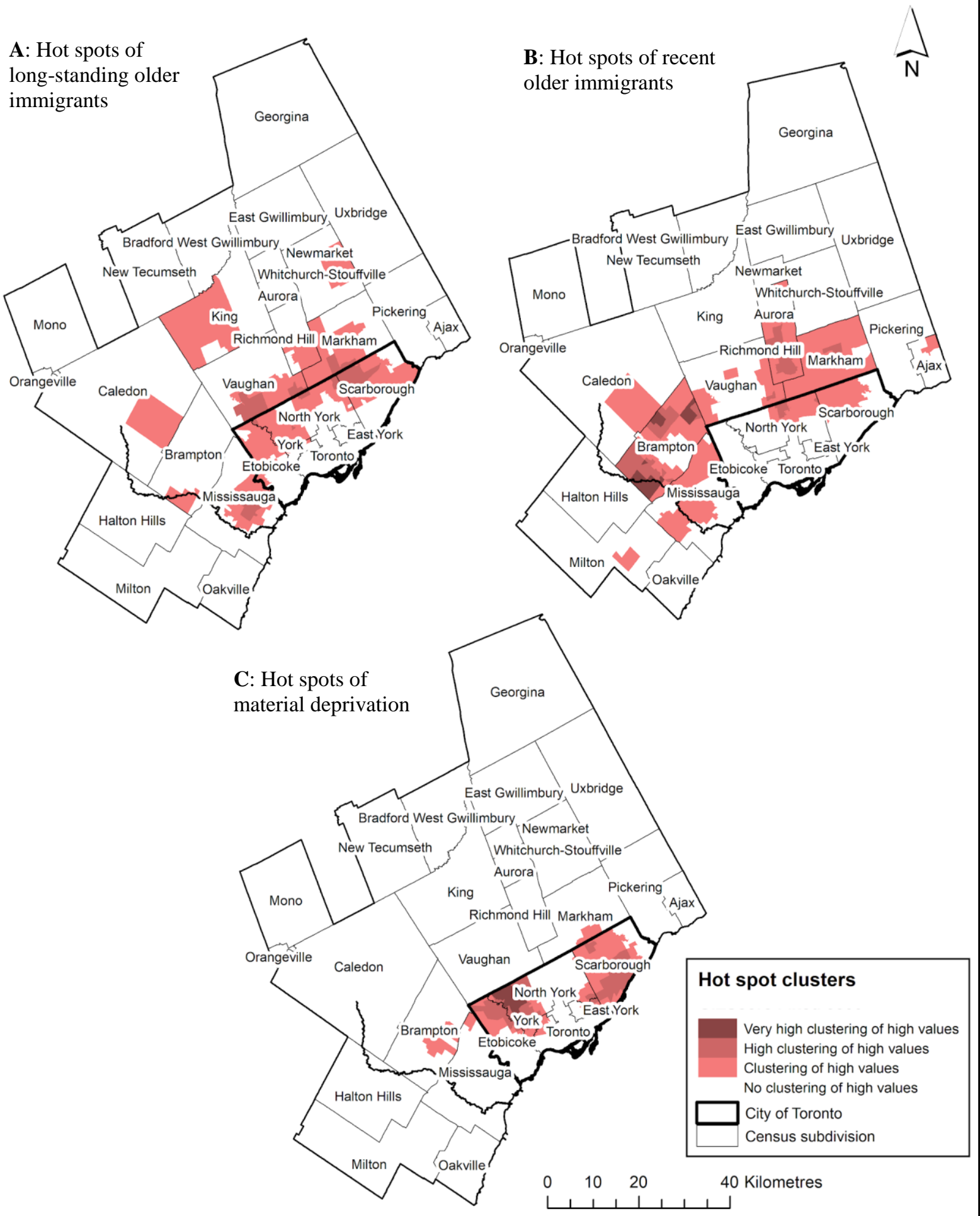

Figure 5: Settlement pattern hot spots for long-standing (a) and recent older immigrants (b), and material deprivation hot spots (c), Toronto CMA, 2016. 
Figures 5 and 6 illustrated the settlement patterns of two groups, long-standing and recent older immigrants. Figures 6a through 6e illustrate the settlement patterns of all older immigrants across the five landing periods that comprised the long-standing and recent groups to present the ten-year interval settlement patterns.

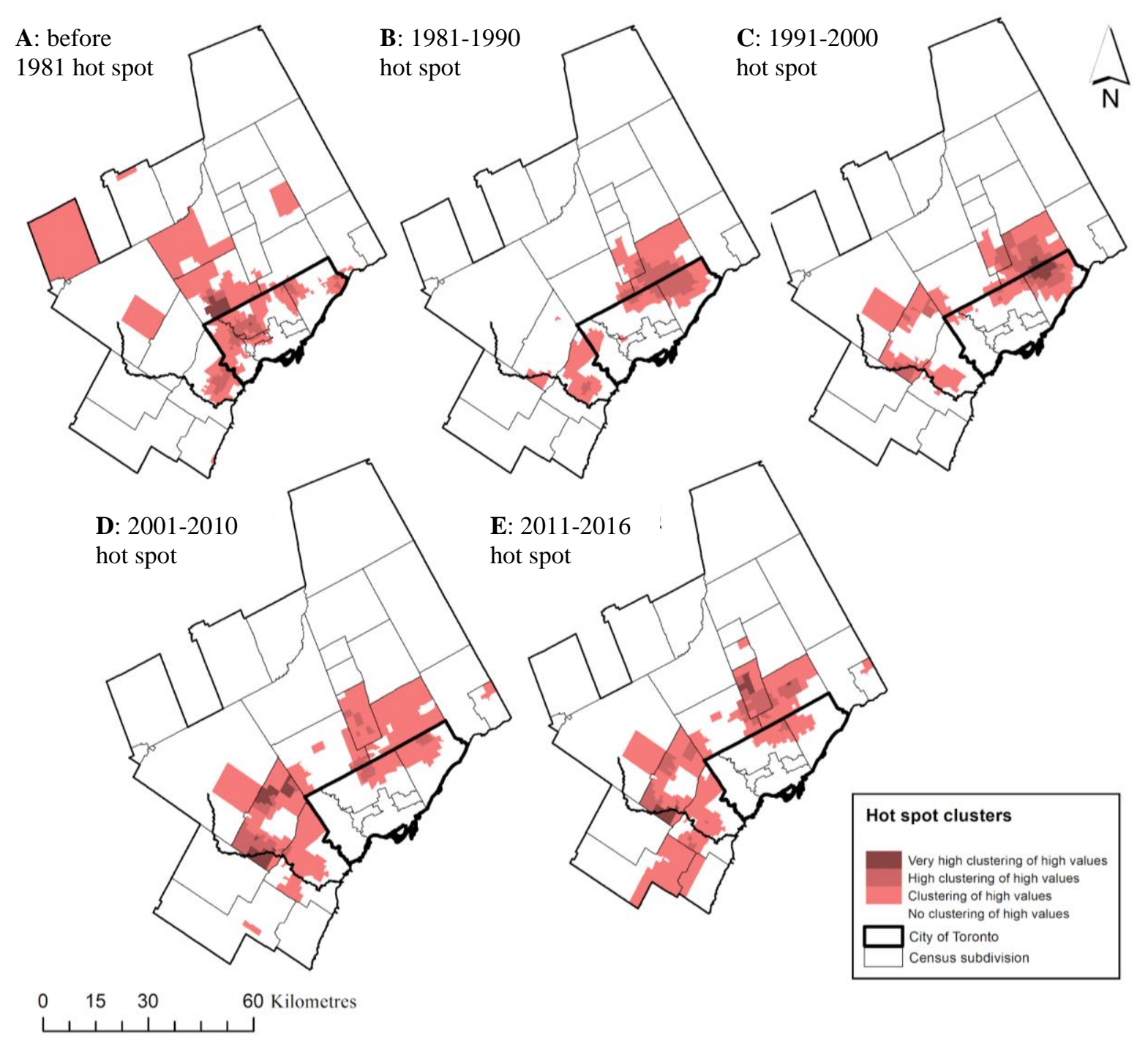

Figure 6: Hot spot of older immigrant settlement during five immigration periods from before 1981 to 2016, Toronto CMA.

Older immigrants that landed in the Toronto CMA before 1981 (Figure 6a) settled predominantly in inner urban neighbourhoods of Toronto and throughout the peripheral municipalities of Vaughan, King and Mono. The inner urban concentrations are found in Toronto's Port Union and Guildwood regions, in the Malvern and Agincourt neighbourhoods, throughout the western-most third of Toronto and north through Woodbridge and Concord. Clusters are also found in central Mississauga, east of the Credit 
River.

Figure 6b depicts the settlement patterns of older immigrants that landed between 1981 and 1990. The spatial distribution of clusters varies significantly from the pre-1981 older immigrants, with two predominant clusters observed. The largest begins north of Highway 401 within Toronto and moves north through most of Markham and Richmond Hill. The second cluster is located in Mississauga, overlapping with the pre-1981 cluster, but spanning northwest through Milton.

Settlement between 1991 and 2000 (Figure 6c) saw similar patterns to that of the previous landing period, but observed higher concentrations of highly clustered regions along the ScarboroughMarkham boundary and spanned further south towards the Toronto lakeshore. The clusters observed previously in Mississauga moved north along the Credit River, through Brampton's west end, and also separately, in north-eastern Brampton along its border with Vaughan.

As shown in Figure 6d, the immigrant settlement patterns between 2001 and 2010 still maintain a similar pattern, however, higher concentrations of statistically significant clustering can be observed throughout all of Brampton (with the exception of the Bramalea area). The large cluster present in the east end of Toronto-Markham appears to be less concentrated. For the first time, there are small clusters in new regions of the CMA, namely north Ajax, Maple (in Vaughan) and in central Milton.

Lastly, the most recent landing period (2011-2016), can be seen in Figure 6e, which differs from the previous settlement distributions. Settlement in the Toronto-Markham cluster appears present, although the cluster itself appears to be smaller. There is, however, a higher concentration of statistically significant cluster CTs in the northern part of Richmond Hill. The cluster in the west end of the CMA is still present and resembles the same pattern as observed in the 2001-2011 distribution except that it appears to be moving westerly, connecting clustering areas in central Mississauga, Oakville and Milton. Higher concentrations of clusters are also observed on both banks of the Credit River when it passes through Brampton. Clustering in northern Ajax also still remains.

\subsection{Group-specific socioeconomic, demographic dimensions and settlement patterns}

\subsubsection{General variation in select socioeconomic and demographic dimensions}

Table 4 provides insights into the socioeconomic and ethnocultural differences and similarities for the older immigrant Chinese, Italian, Portuguese and South Asian populations.

Proportions for the age-based characteristics tells us that only the Italian older immigrants have more individuals aged 75+ than they do aged 65-74. Chinese, Portuguese and South Asians have a higher proportion of immigrant individuals aged 65-74 years compared to their 75+ age group. Of the total older immigrant population in Toronto, $12 \%$ are South Asian, $11 \%$ are Chinese and $9.2 \%$ are Italian, while only $4 \%$ are Portuguese. Of each ethnocultural immigrant population the share that are male or female is comparable across the four groups, with a higher proportion of females than males for each. 
Table 4: Comparing group-specific socioeconomic and demographic compositions.

\begin{tabular}{|c|c|c|c|c|c|c|c|c|c|c|c|c|c|c|c|c|}
\hline \multirow{2}{*}{ Dimension } & \multicolumn{4}{|c|}{$\begin{array}{l}\text { Chinese older } \\
\text { immigrants }\end{array}$} & \multicolumn{4}{|c|}{$\begin{array}{l}\text { Italian older } \\
\text { immigrants }\end{array}$} & \multicolumn{4}{|c|}{$\begin{array}{l}\text { Portuguese older } \\
\text { immigrants }\end{array}$} & \multicolumn{4}{|c|}{$\begin{array}{l}\text { South Asian older } \\
\text { immigrants }\end{array}$} \\
\hline & $\#$ & $\begin{array}{l}\% \\
\text { older } \\
\text { pop }\end{array}$ & $\begin{array}{l}\% \\
\text { imm } \\
\text { older } \\
\text { pop }\end{array}$ & $\begin{array}{l}\% \text { sub } \\
\text { group }\end{array}$ & $\#$ & $\begin{array}{l}\% \\
\text { older } \\
\text { pop }\end{array}$ & $\begin{array}{l}\% \\
\text { imm. } \\
\text { older } \\
\text { pop }\end{array}$ & $\begin{array}{l}\% \text { sub } \\
\text { group }\end{array}$ & $\#$ & $\begin{array}{l}\% \\
\text { older } \\
\text { pop }\end{array}$ & $\begin{array}{l}\% \\
\text { imm. } \\
\text { older } \\
\text { pop }\end{array}$ & $\begin{array}{l}\% \text { sub } \\
\text { group }\end{array}$ & $\#$ & $\begin{array}{c}\% \\
\text { older } \\
\text { pop }\end{array}$ & $\begin{array}{l}\% \\
\text { imm. } \\
\text { older } \\
\text { pop }\end{array}$ & $\begin{array}{l}\% \text { sub } \\
\text { group }\end{array}$ \\
\hline \multicolumn{17}{|c|}{ Age and gender } \\
\hline $\begin{array}{l}65+ \\
\text { population }\end{array}$ & 88,965 & 10.9 & 15.3 & 100 & 74,635 & 9.2 & 12.8 & 100 & 28,535 & 3.5 & 4.9 & 100 & 96,645 & 11.9 & 16.6 & 100 \\
\hline $\begin{array}{l}65-74 \text { years } \\
\text { old }\end{array}$ & 50,945 & 10.9 & 8.8 & 57.3 & 35,570 & 9.2 & 6.1 & 47.7 & 16,505 & 3.5 & 2.8 & 57.8 & 62,155 & 11.9 & 10.7 & 64.3 \\
\hline $75+$ & 38,015 & 0 & 6.5 & 42.7 & 39,065 & 0 & 6.7 & 52.3 & 12,030 & 0 & 2.1 & 42.2 & 34,490 & 0 & 5.9 & 35.7 \\
\hline Male & 40,445 & 5.0 & 7.0 & 45.5 & 35,775 & 4.4 & 6.2 & 47.9 & 12,735 & 1.6 & 2.2 & 44.6 & 47,030 & 5.8 & 8.1 & 48.7 \\
\hline Female & 48,515 & 5.9 & 8.3 & 54.5 & 38,860 & 4.8 & 6.7 & 52.1 & 15,795 & 1.9 & 2.7 & 55.4 & 49,625 & 6.1 & 8.5 & 51.3 \\
\hline \multicolumn{17}{|l|}{ Income } \\
\hline Low & 57,685 & 7.1 & 9.9 & 65 & 19,940 & 2.5 & 3.4 & 27 & 10,790 & 1.3 & 1.9 & 38 & 61,960 & 7.6 & 10.7 & 64 \\
\hline Medium & 19,265 & 2.4 & 3.3 & 22 & 38,925 & 4.8 & 6.7 & 52 & 13,815 & 1.7 & 2.4 & 48 & 21,240 & 2.6 & 3.7 & 22 \\
\hline High & 10,830 & 1.3 & 1.9 & 12 & 12,785 & 1.6 & 2.2 & 17 & 3,345 & 0.4 & 0.6 & 12 & 12,400 & 1.5 & 2.1 & 13 \\
\hline \multicolumn{17}{|c|}{ Education attainment } \\
\hline No education & 30,105 & 3.7 & 5.2 & 33.8 & 48,685 & 6.0 & 8.4 & 65.2 & 18,825 & 2.3 & 3.2 & 66.0 & 32,325 & 4.0 & 5.6 & 33.4 \\
\hline $\begin{array}{l}\text { Secondary } \\
\text { education }\end{array}$ & 24,060 & 3.0 & 4.1 & 27.0 & 9,870 & 1.2 & 1.7 & 13.2 & 4,845 & 0.6 & 0.8 & 17.0 & 23,900 & 2.9 & 4.1 & 24.7 \\
\hline $\begin{array}{l}\text { Post- } \\
\text { secondary } \\
\text { education }\end{array}$ & 34,795 & 4.3 & 6.0 & 39.1 & 16,090 & 2.0 & 2.8 & 21.6 & 4,855 & 0.6 & 0.8 & 17.0 & 40,435 & 5.0 & 7.0 & 41.8 \\
\hline \multicolumn{17}{|c|}{ Language spoken most at home } \\
\hline English & 10,160 & 1.2 & 1.7 & 11.4 & 22,520 & 2.8 & 3.9 & 30.2 & 8,020 & 1 & 1.4 & 28.1 & 32,065 & 3.9 & 5.5 & 33.2 \\
\hline French & 55 & 0 & 0 & 0.1 & 95 & 0 & 0 & 0.1 & 25 & 0 & 0 & 0.1 & 70 & 0.0 & 0 & 0.1 \\
\hline $\begin{array}{l}\text { Non-official } \\
\text { language }\end{array}$ & 73,810 & 9.1 & 12.7 & 83 & 43,515 & 5.3 & 7.5 & 58.3 & 18,590 & 2.3 & 3.2 & 65.1 & 53,220 & 6.5 & 9.2 & 55.1 \\
\hline $\begin{array}{l}\text { English and } \\
\text { French }\end{array}$ & 15 & 0 & 0 & 0 & 45 & 0 & 0 & 0.1 & 0 & 0 & 0 & 0 & 35 & 0.0 & 0 & 0 \\
\hline $\begin{array}{l}\text { English and } \\
\text { non-official } \\
\text { language }\end{array}$ & 4,870 & 0.6 & 0.8 & 5.5 & 8,350 & 1 & 1.4 & 11.2 & 1,875 & 0.2 & 0.3 & 6.6 & 11,200 & 1.4 & 1.9 & 11.6 \\
\hline \multicolumn{17}{|c|}{ Period of immigration } \\
\hline before 1981 & 30,085 & 3.7 & 5.2 & 33.8 & 72,365 & 8.9 & 12.4 & 97 & 23,870 & 2.9 & 4.1 & 83.7 & 32,460 & 4 & 5.6 & 33.6 \\
\hline $1981-1990$ & 18,535 & 2.3 & 3.2 & 20.8 & 1,265 & 0.2 & 0.2 & 1.7 & 2,810 & 0.3 & 0.5 & 9.8 & 13,805 & 1.7 & 2.4 & 14.3 \\
\hline $1991-2000$ & 22,395 & 2.7 & 3.9 & 25.2 & 540 & 0.1 & 0.1 & 0.7 & 1,275 & 0.2 & 0.2 & 4.5 & 24,945 & 3.1 & 4.3 & 25.8 \\
\hline $2001-2010$ & 10,100 & 1.2 & 1.7 & 11.4 & 365 & 0 & 0.1 & 0.5 & 395 & 0 & 0.1 & 1.4 & 18,080 & 2.2 & 3.1 & 18.7 \\
\hline 2011-2016 & 7,840 & 1.0 & 1.3 & 8.8 & 105 & 0 & 0 & 0.1 & 175 & 0. & 0 & 0.6 & 7,370 & 0.9 & 1.3 & 7.6 \\
\hline
\end{tabular}


Income distributions of the four ethnocultural subgroups are provided in Figure 7. It is evident that there is a large income gap between the long-standing and recent immigrant groups. Italian older immigrants appear to be the wealthiest as they have the lowest proportions of low income, and the highest proportions of medium and high income $(27 \%, 52 \%$ and $17 \%$, respectively), followed by Portuguese (38\%, 48\%, 12\%, respectively). Comparatively, the Chinese and South Asian older immigrants exhibit similar patterns, with the highest rates of low income, and the lowest rates of medium and high income. Almost two-thirds of the older immigrant Chinese and South Asian populations are low income earners (65\% and 64\%, respectively). In the high income class, it is evident that the income gap is smaller, as the proportions of each subgroup that earn more than $\$ 50,000$ only range from $12 \%-17 \%$.

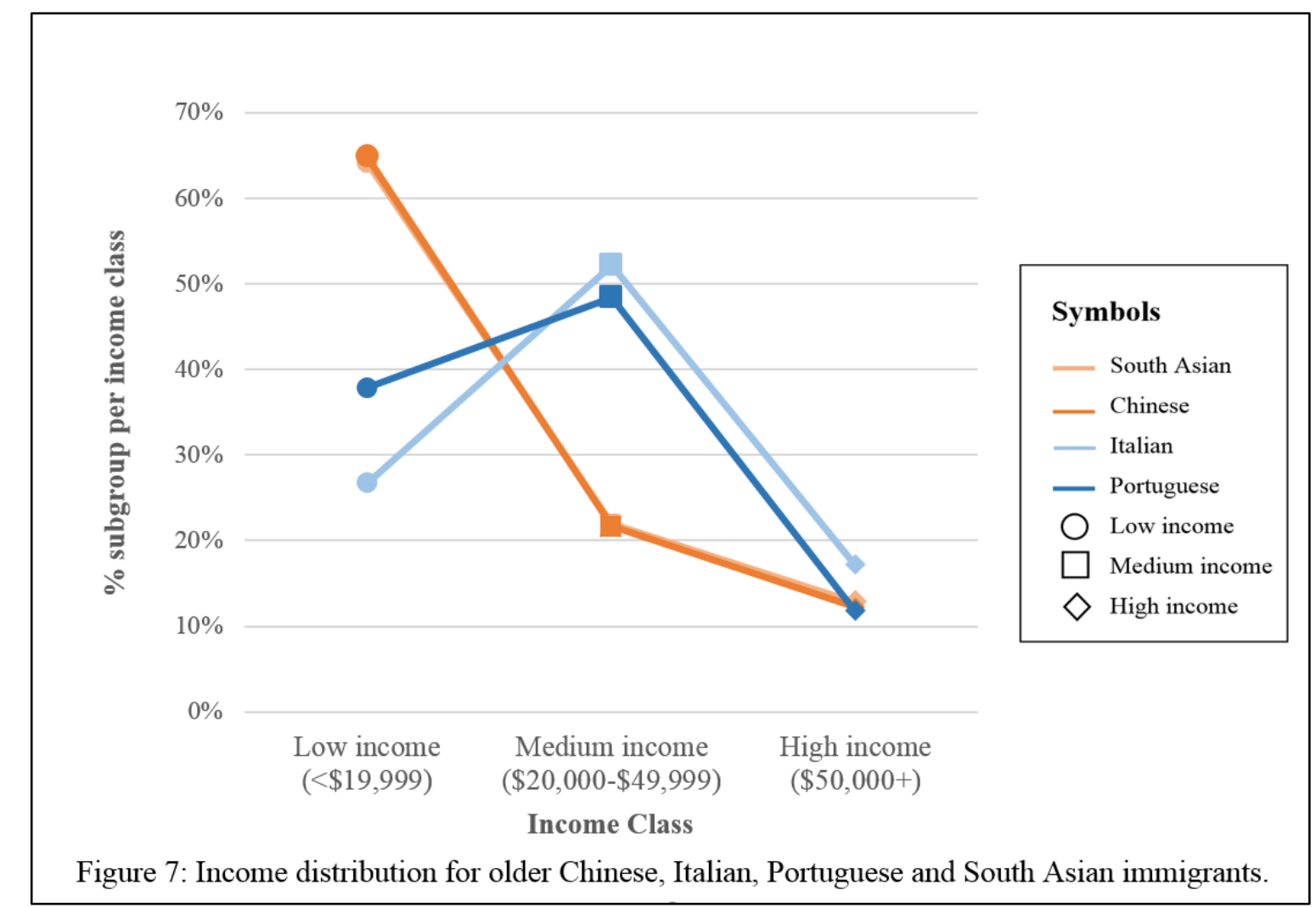

Figure 8 illustrates education attainment for the four subgroups. South Asian older immigrants observe the highest proportion of post-secondary education attainment (42\%) and second highest high school education attainment (25\%) when compared to the other three ethnocultural groups, meaning they have the highest proportion of highly educated older immigrants. Conversely, the Italian and Portuguese older immigrants appear to be the least educated, where $65 \%$ and $66 \%$ them, respectively, have no formal education and $22 \%$ and $17 \%$, respectively, have achieved post-secondary degrees. 


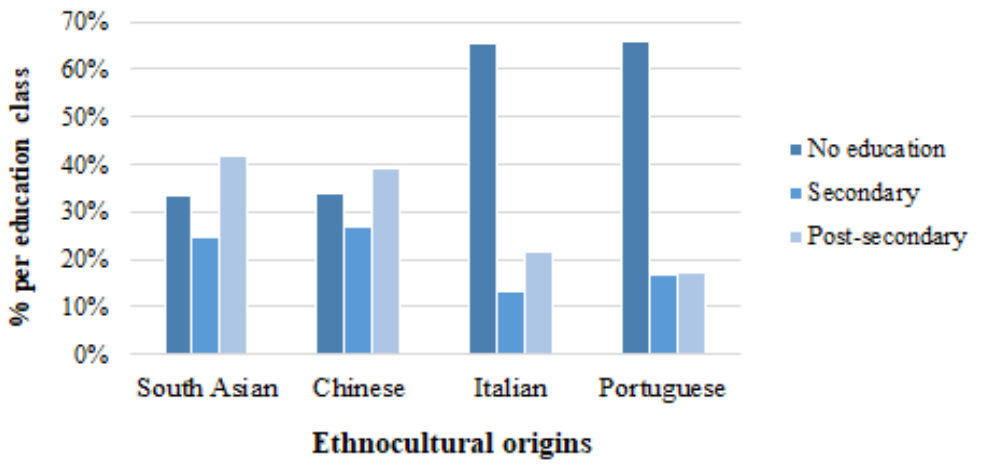

Figure 8: Proportion of each ethnocultural group per education class.

The ethnocultural characteristics of the four study population presented in Table 4 also indicate variations amongst the groups. In terms of language most spoken at home, Chinese older immigrants appear to have the highest share of non-official language-speaking older immigrants (83\%), whereas Portuguese, Italian and South Asian subgroups appear to have significantly lower shares in the same language category $(65 \%, 58 \%$ and $55 \%$, respectively). The ethnocultural group with the highest share of English-speaking older immigrants are South Asian, where 33\% of their subgroup speaks English, and $11.6 \%$ of this subgroup speaks both English and a non-official language most often at home. Portuguese and Italian subgroups appear to have similar adoption of language preferences, where $28 \%$ and $30 \%$ of their study populations speak English only. For all ethnocultural groups, less than $0 \%$ of their study populations speak both English and French only.

Regarding period of immigration, each ethnocultural subgroup exhibits different migration patterns based on the years in which they migrated. It appears that the Italian and Portuguese older immigrant populations are the most long-standing as they have the highest share of their subgroup arriving before 1981, as shown in Table 4. A staggering 97\% of Italian older immigrants and $84 \%$ of Portuguese arrived in this period. Comparatively, Chinese and South Asian older immigrants appear to have higher proportions of recently arrived immigrants in the 2001-2010 (11\% and 19\%, respectively) and in the 2011-2016 landing periods (9\% and 8\%, respectively). Conversely, both the Portuguese and Italian older immigrants that arrived in those recent landing periods comprised a share of less than $1 \%$ of their subgroups.

\subsubsection{Education, landing period and language's influence on income}

Given that the data provided was cross-tabular in form, evaluating the subgroup populations using multiple layers of socioeconomic data can provide insight into the influence that language, education 
attainment and landing period might appear to have on income. Figure 9 illustrates these variable relationships and indicates the proportion of each subgroup population that fall into a language class, based on their income.

As shown in Figure 9a, the relationship between education and attainment for each ethnocultural older immigrant subgroup is not the same. Overall, the chart suggests that higher education results in higher income, but the in-group proportions vary dramatically. Across all three education levels, there is a large gap in the low income class between more recent subgroups (Chinese and South Asian older immigrants) and long-standing subgroups (Italian and Portuguese older immigrants). 80\% of Chinese older immigrants and $86 \%$ of South Asian older immigrants with no education earn less than $\$ 19,999$, in comparison to values half as large for Italian and Portuguese subgroups (30\% and 42\%, respectively). The income gap for medium income class in the no education category is similarly large. When examining the income distribution for older immigrants with post-secondary degrees, the gaps are not as prevalent, but Italian and Portuguese subgroups still perform better than their recent subgroup counterparts. Exactly half of the Chinese older immigrant population and $44 \%$ of the South Asian subgroup who have a postsecondary degree earn less than $\$ 19,999$. Almost half of both Italian and Portuguese older immigrants with post-secondary degrees are in the medium income class ( $46 \%$ and $49 \%$, respectively). Lastly, Italians fare the best in the high income class for those with post-secondary degrees (37\%), followed by $29 \%$ of the Portuguese subgroup, 24\% of the South Asian subgroup and 23\% of the Chinese older immigrant subgroup.

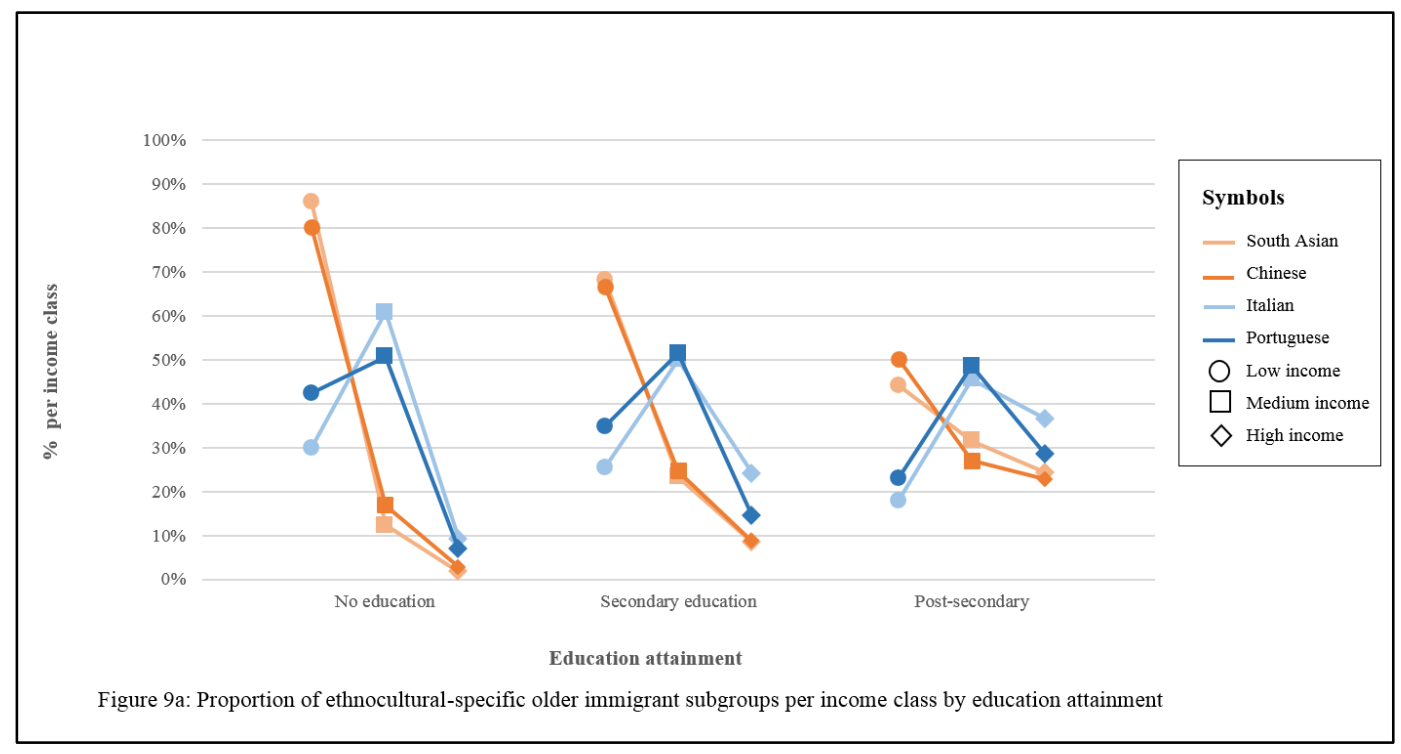

The relationships between language spoken most often at home and income class reveal interesting results. As shown in Figure 9b, long-standing immigrants perform better in income classes in almost all language categories, with the largest income gaps in the non-official language category. For 
older immigrants that speak mostly an official language at home, the proportions across all four ethnocultural subgroups are relatively comparable, but most notably, English and French speaking Chinese and South Asian older immigrants are proportionately higher in the low income class and the marginally higher in the high income class than the Italian and Portuguese counterparts. However, these two recent older immigrant groups suffer from lack of knowledge of an official language, where $79 \%$ and $72 \%$ of South Asian and Chinese older immigrants, respectively are low income earners. Interestingly, even for the older immigrant subgroups that speak English and a non-official language most at home, the South Asian and Chinese subgroups have depressed income (55\% and 38\% low income earners, respectively), yet they also perform marginally better in the high income class when compared to their Italian and Portuguese counterparts.

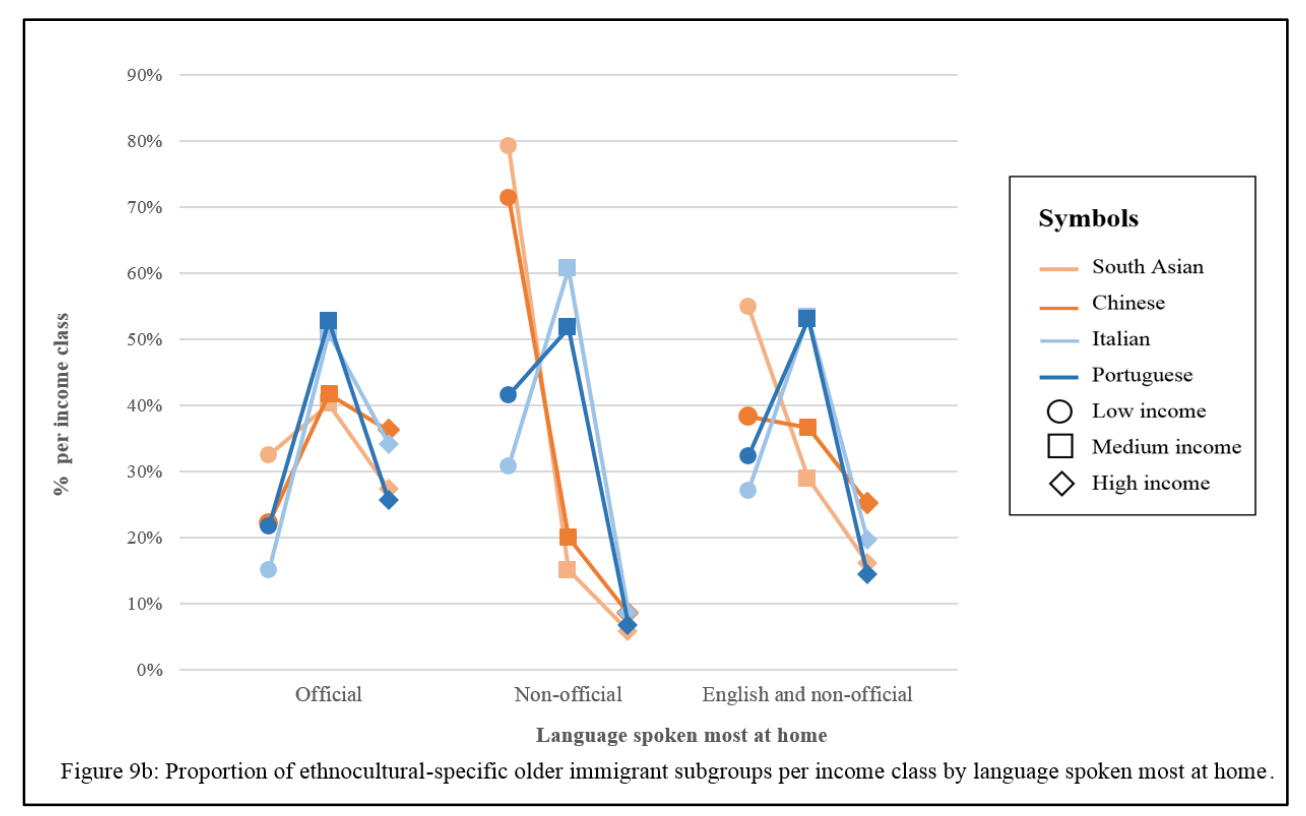

Income and landing period were also charted, as presented in Figure 9c. The data depicts a pattern that suggests that income distribution for long-standing immigrant groups is more stable, with marginal gaps. Conversely, for recent older immigrants (in the 2001-2010 landing period class and the 2011-2016 landing period class), particularly the Chinese and South Asian subgroups, there is an alarmingly high prevalence of low income. All of the subgroups observed their highest share of high income for those that landed before 1981, and all of the subgroups saw a subsequent decline in high income attainment. For the Chinese and South Asian subgroups the decline was dramatic, while the Italians and Portuguese exhibited a gradual decline. Additionally, every ten-year interval observed higher proportions of lower income for all subgroups, and most notably, the recent subgroups (Chinese and South Asian) appear to have the most depressed income. For the Chinese and South Asian older immigrants that landed between 2001 and 2010, 93\% and 87\% exhibited low income, respectively, and in the 2011-2016 landing period, 96\% and 
$94 \%$ of them were low income earners, respectively. This is a contrast in comparison to the significantly smaller proportions of low income earning Italian and Portuguese subgroups for these landing periods.

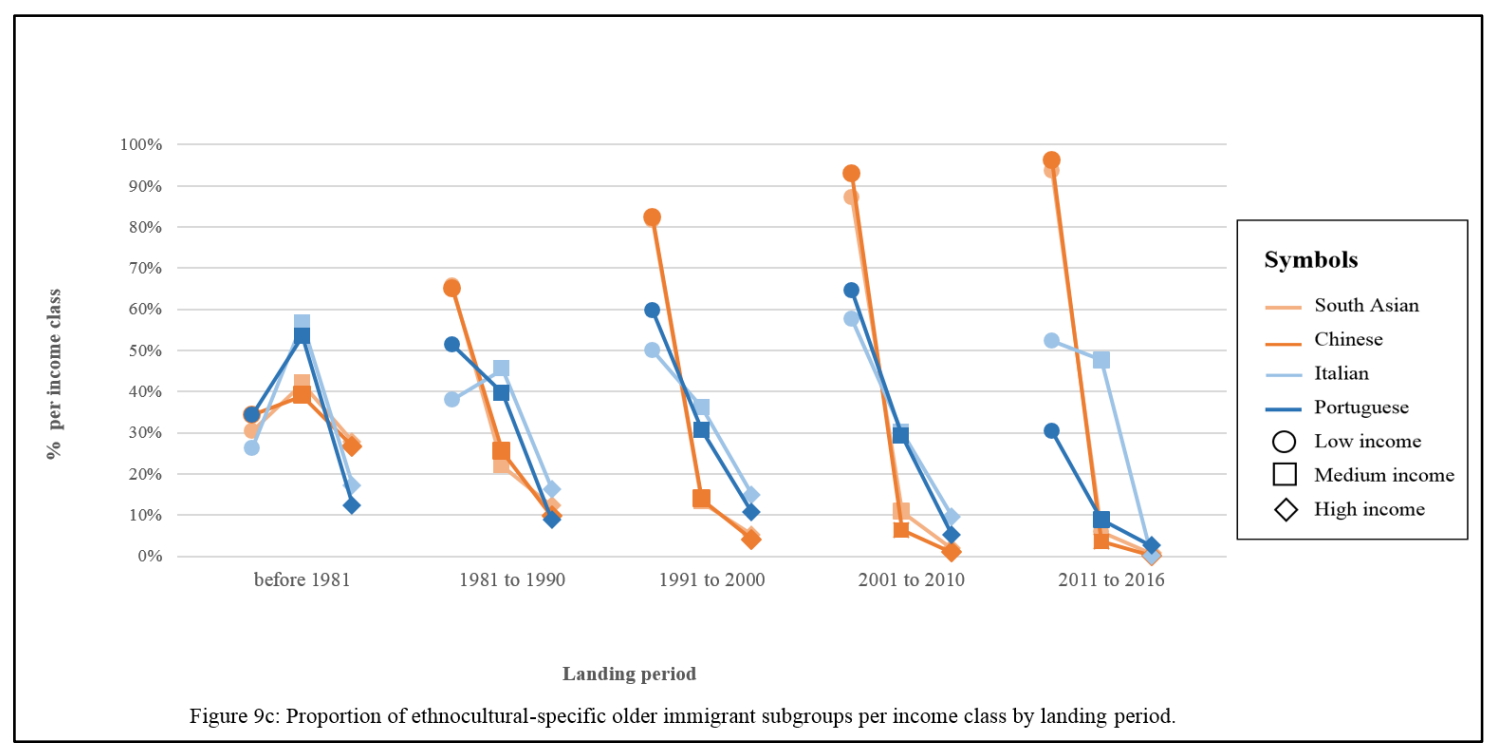

Lastly, Table 5 depicts the share of each older immigrant subgroup based on their immigration period category, suggesting that the older Italian and Portuguese immigrants are largely characterized by their established residency in the Toronto CMA (99\% and 98\% arrived before 2000, respectively), while the older Chinese and South Asian immigrants are largely characterized by their recent immigrants in the Toronto CMA (20\% and 26\% arrived after 2001, respectively).

Table 5: Share of each older immigrant ethnocultural subgroup by long-standing or recent categorization.

\begin{tabular}{|l|c|c|c|c|c|c|c|c|}
\hline \multirow{2}{*}{ Landing period } & \multicolumn{2}{|c|}{ Chinese } & \multicolumn{2}{|c|}{ Italian } & \multicolumn{2}{c|}{ Portuguese } & \multicolumn{2}{c|}{ South Asian } \\
\cline { 2 - 9 } & $\#$ & $\begin{array}{c}\% \\
\text { subgroup }\end{array}$ & $\#$ & $\begin{array}{c}\% \\
\text { subgroup }\end{array}$ & $\#$ & $\begin{array}{c}\% \\
\text { subgroup }\end{array}$ & $\#$ & $\begin{array}{c}\% \\
\text { subgroup }\end{array}$ \\
\hline $\begin{array}{l}\text { Long-standing (before } \\
2000)\end{array}$ & 71,015 & $80 \%$ & 74,170 & $99 \%$ & 27,955 & $98 \%$ & 71,210 & $74 \%$ \\
\hline Recent (2001-2016) & 17,940 & $20 \%$ & 470 & $1 \%$ & 570 & $2 \%$ & 25,450 & $26 \%$ \\
\hline
\end{tabular}

\subsubsection{Group-specific settlement patterns}

Overall, each ethnocultural subgroup appears to have their own set of statistically significant clusters independent of each other, with the exception of Chinese and South Asian older immigrant clusters which overlap in some instances. Figure 10 presents the relative representation of each ethnocultural subgroup in the CMA using Location Quotient. Figure 10a shows Chinese older immigrants per CT relative to their concentration in the entire CMA. There is under representation through most of the rural peripheral municipalities, like northern Uxbridge, Pickering, Ajax, New Tecumseth, Caledon, Brampton, Oakville, parts of Mississauga, and the western half of Toronto. In areas where there is over representation of older Chinese immigrants, CTs of moderate representation are typically observed to be close in proximity. 
Overrepresentation of this subgroup is located in the downtown Toronto core, along Dundas Street and Spadina Avenue (which includes Chinatown), and moving north through York and Downsview. There is also an overrepresentation in the eastern half of Vaughan, parts of Richmond Hill, and Markham. CTs of extreme overrepresentation, meaning there is at least 5.2 times the amount of Chinese older immigrants in that particular CT than there is in the entire CMA, are found in Chinatown, in the Steeles Avenue and Victoria Park Avenue area, and in three CTs within Richmond Hill.

Older Italian immigrant concentrations can be seen in Figure 10b where a large region of CTs with over and extremely overrepresentation of this subgroup can be observed beginning adjacent to the downtown Toronto core (Little Italy) and in a V-shaped trajectory northerly to the upper boundaries of the CMA. This V-shaped area of high concentration encompasses Vaughan, King, parts of Caledon, and West Gwillimbury. Within this region, the areas of extreme overrepresentation are found in Maple, Concord, and Kleinburg, and down in Toronto's neighbourhoods of Mt Dennis, Weston, and Eglinton West. Whitchurch-Stouffville, the northern part of Markham and Pickering, also observe over to moderately overrepresentation. Scattered CTs throughout the rest of the urban and suburban regions also exhibit overrepresentation, while rural municipalities on the western and eastern flanks of the CMA exhibit underrepresentation.

As shown in Figure 10c, older Portuguese immigrant concentrations are not as expansive as the other ethnocultural groups and are isolated into a very small segment of the CMA. Areas that observe extreme overrepresentation (surrounded closely by areas of over and moderate representation) can be observed adjacent to the downtown Toronto core beginning in Little Portugal, moving through Parkdale, and then north through High Park, The Junction, and Eglinton West. Scattered CTs of moderate representation areas are also observed in southern Etobicoke and throughout the southern half of Mississauga. Small pockets of moderate and over represented Italian older immigrants are also found in Brampton's Bramalea area, in southern Oakville, and in Bradford West Gwillimbury.

As can be seen in Figure 10d, the spatial distribution of older South Asian immigrant concentrations is predominantly located in suburban regions to the west of the CMA and inner urban regions to the east. The region on the east includes CTs with moderate and over representation of South Asian older immigrants, and this area encompasses most of Scarborough, down to the Toronto lakeshore and north through the neighbourhoods in Markham that boundary Toronto. North Ajax also observes CTs with moderate and over representation of this subgroup. Scattered CTs of moderate and over representation are observed in other parts of Markham, Richmond Hill and Vaughan, and in parts of Oakville and Milton. A large area in Mississauga and Brampton exhibits an overrepresentation of South Asian immigrants, and in a few CTs, an extremely over representation. 


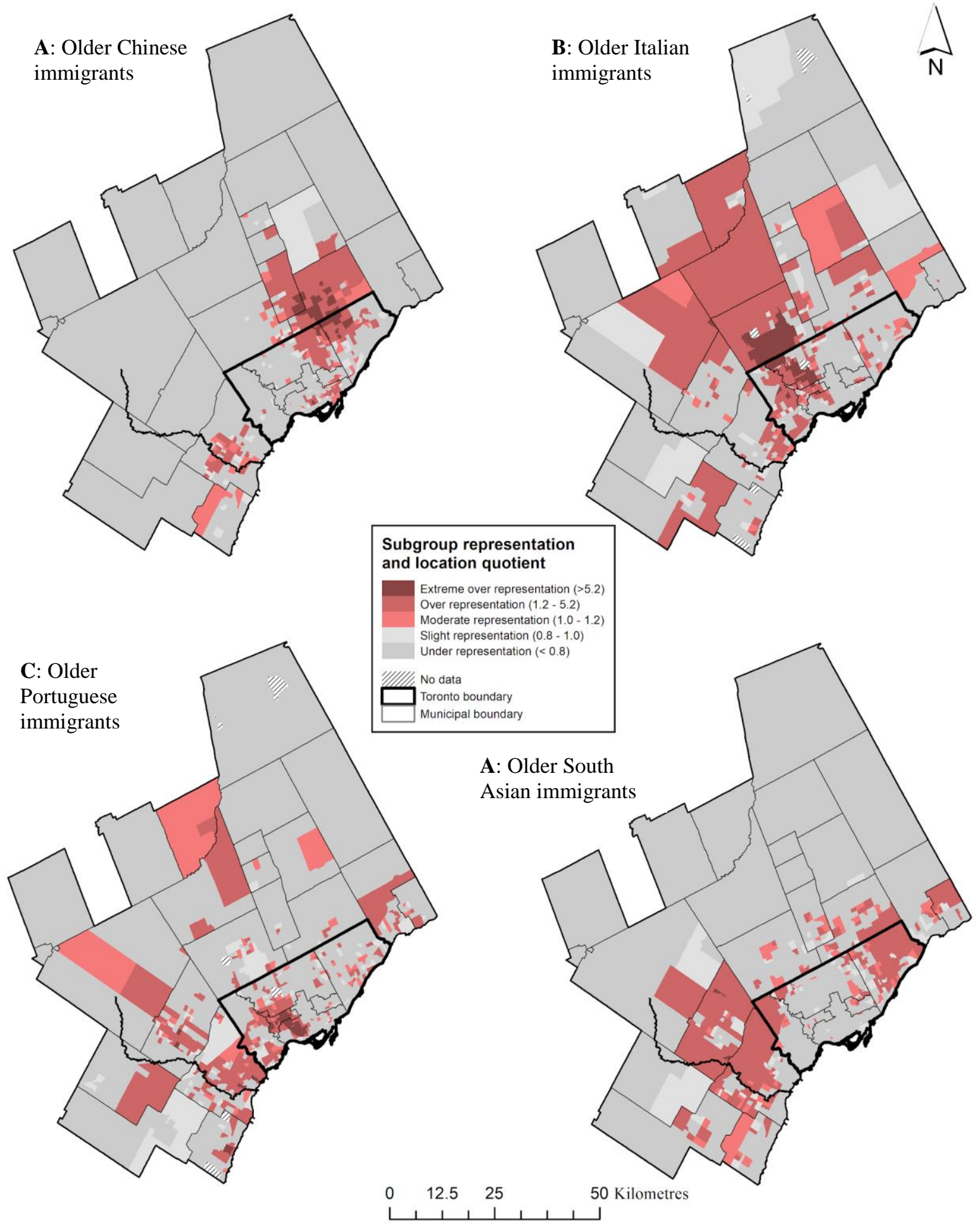

Figure 10: LQ of ethnoculturally-specific older immigrant subgroups, Toronto CMA, 2016. 


\subsubsection{Variation in group-specific settlement patterns for long-standing and recent subgroups}

Long-standing older Chinese immigrants, arriving before the year 2000, appear to have settled in one large cluster in the Toronto CMA (Figure 11a) located along the Toronto-Markham border, encompassing most of Markham and into Richmond Hill. Areas with the highest statistical significance and confidence can be found in Malvern, Agincourt, Steeles, Unionville, and Greensborough, through parts of southeastern Richmond Hill. Older recent Chinese immigrants appear in a similar pattern, but do not have clusters with such high intensity as their long-standing counterparts (Figure 11b). In addition, a small cluster of recent Chinese older immigrants can be found in Brampton, and along the Aurora-Newmarket border.

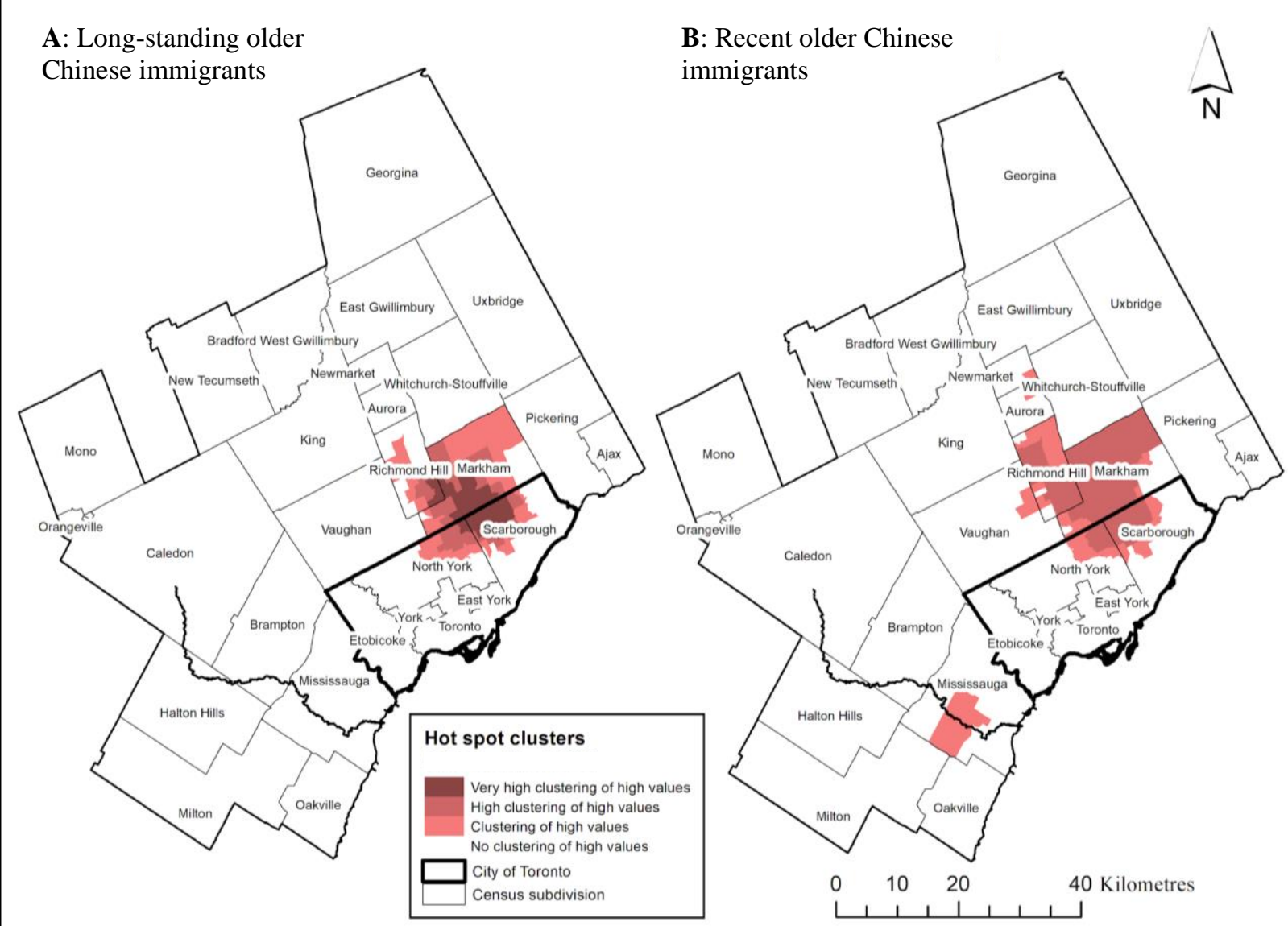

Figure 11: Long-standing and recent Chinese older immigrant settlement hot spots, Toronto CMA, 2016.

As illustrated in Figure 12a, long-standing older Italian immigrants have settled in one large cluster in the Toronto CMA, located in north-western Toronto, and travelling north through Vaughan, King and parts of Caledon. There are instances of high clustering of high values within the heart of this cluster, specifically in the Weston, Eglinton West, Maple, and Concord areas. Conversely, recent older Italian immigrant settlement patterns are markedly different than their long-standing counterparts (Figure $12 \mathrm{~b})$. Although there is overlap in the high concentration of clusters in most of Vaughan, there is also 
clustering now spread throughout other peripheral municipalities, namely Aurora, Newmarket, Richmond Hill, Whitchurch-Stouffville, parts of Toronto, and throughout Mississauga's northern half, most of Oakville, and the southern part of Milton.

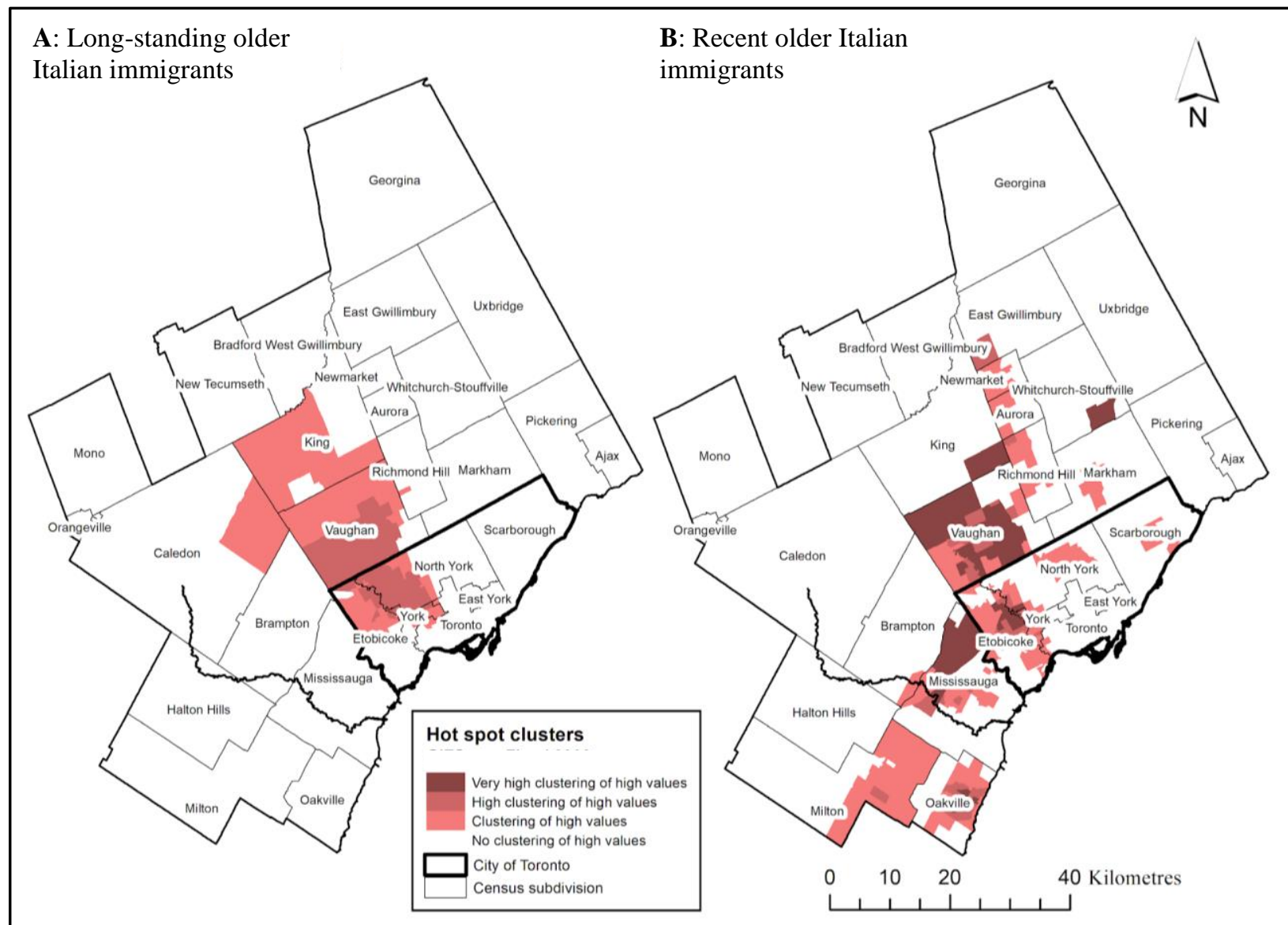

Figure 12: Long-standing and recent Italian older immigrant settlement hot spots, Toronto CMA, 2016.

Long-standing older South Asian immigrants appear to have relatively similar settlement patterns as their recent counterparts, with a few slight differences. As shown in Figure 13a, the long-standing South Asian older immigrant population has two large clusters of high values; one located around Scarborough and Markham, and the other located in Brampton, Mississauga, and in parts of Vaughan and north-western Toronto. There is also a small cluster of located in north-eastern Ajax. Figure 13b exhibits the settlement patterns of the recent cohort, and despite the clusters appearing to overlap with their longstanding counterparts, there are areas with very high clustering of older South Asian immigrants, particularly within Brampton. Additionally, the long-standing subgroup's eastern cluster, which engulfed all of Scarborough through to the lakefront, has moved north for the recent subgroup, now encompassing most of Markham, areas along the Markham-Vaughan border, and all of Richmond Hill. This cluster goes as far south as Highway 401 in Toronto. The small cluster of long-standing South Asian immigrants that 
was in Ajax is still present but has decreased in size. Additional small clusters of recent older South Asian immigrants are observed in Milton, central Vaughan and northern Newmarket.

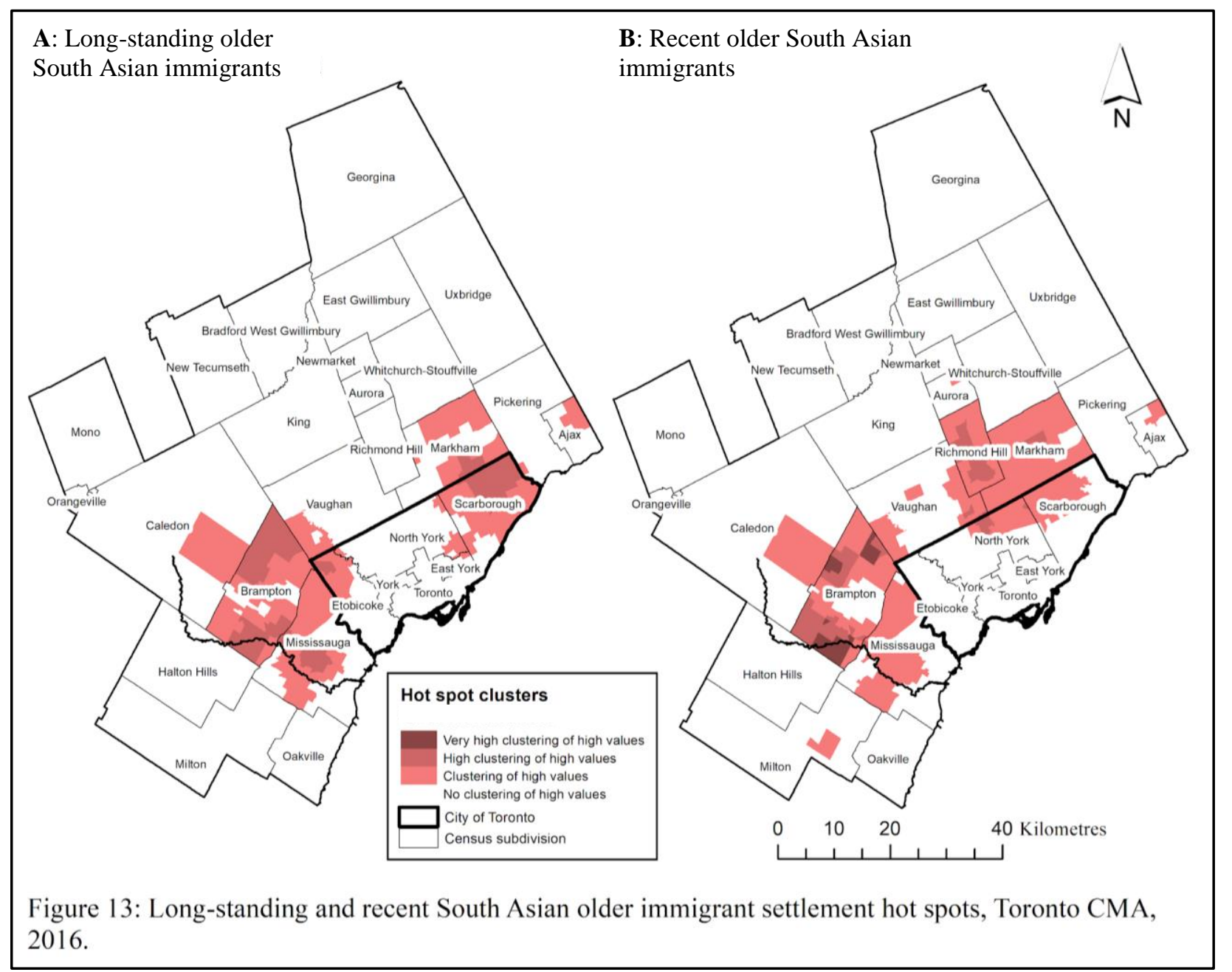

Figure 14a presents the settlement patterns of long-standing older Portuguese immigrants and are compared to the settlement patterns of their recent immigrant counterparts (Figure 14b). There are four clusters of long-standing Portuguese older immigrants in the CMA. The largest and most concentrated with the highest values is located adjacent to the downtown Toronto core, engulfing all of Little Portugal. The second is located in southern Mississauga the third in Bramalea (central Brampton), and the last can be observed in a small pocket of Bradford West Gwillimbury. In stark contrast, recent older Portuguese immigrants exhibit a different settlement pattern, with numerous pockets of clustering throughout the southern half of the CMA. Clustering adjacent to the downtown Toronto core, in Little Portugal and clustering in Mississauga and Brampton still remains, but those clusters have expanded in size. Additional clusters are observed throughout most of Ajax, in the Malvern area, the Beaches neighbourhood, Maple, and in a small pocket within Milton. 


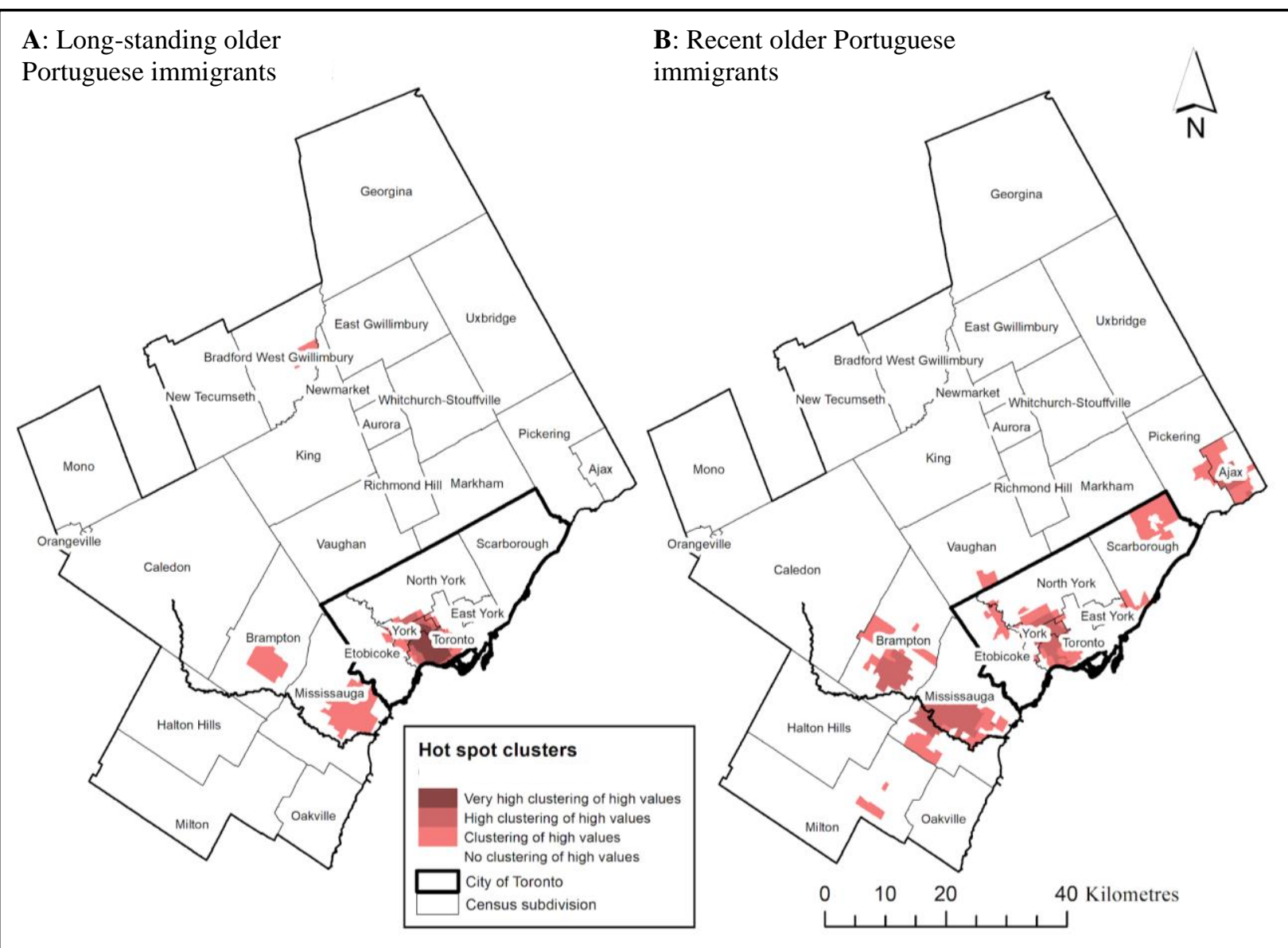

Figure 14: Long-standing and recent Portuguese older immigrant settlement hot spots, Toronto CMA, 2016.

\section{Discussion and Conclusions}

\subsection{Summary of findings}

The majority of this research paper's findings are in support of the relevant, albeit limited, literature on older immigrant population composition and spatial distribution. Almost three-quarters of the older population in the Toronto CMA are immigrants who hold the highest rates of low income in comparison to their non-immigrant counterparts. Education and language are influential factors in income earning potential. However, devaluation of labour and foreign-achieved education depresses employment opportunities and income generation. There are consistent income gaps between older immigrants and older non-immigrants, and across the ethnocultural-specific subgroups, predominantly dictated by the recent and long-standing classifications. Long-standing older immigrants are more socioeconomically stable than their recent counterparts. Further to Pendakur and Pendakur's (2011), Creese's (2007) and Galabuzi's (2006) research, lack of an official Canadian language was found to significantly depress older immigrant earnings. Recent older immigrants, in particular, are not as fluent in official languages as their 
long-standing counterparts. Unfortunately, this points to the systemic issue of racialization in labour markets in general, and a shift in the way that all levels of Canadian government inform immigrantrelated policy.

\subsection{Discussion of results}

\subsubsection{Variation in socioeconomic and demographic composition}

Older immigrants are not a homogenous group of individuals and as such cannot be provided with a generalized, culturally and linguistically inappropriate collection of settlement services that exacerbate their economic vulnerability, social and cultural prosperity, and adjustment and integration into Canadian society as argued by Nazroo (2006), Phillipson, Alhaq, Ullah and Ogg (2000), and Yu (2000) (as cited by Zubair \& Norris, 2015). Toronto's population is aging and experiencing a shift in ethnocultural diversification, away from Europe and towards Asian sending-countries, hence, settlement services for newcomers should reflect this transition and adapt to the needs of Canada's older immigrants (Statistics Canada, 2013).

Understanding not only the socioeconomic and demographic dimensions of these population groups, but more importantly, where older immigrants decide to settle is a critical component in the planning and delivery of essential services. Consequently, location-specific analyses of ethnoculturalspecific groups is largely absent in recent literature on Canadian immigrants. In particular, as the shift in ethnocultural diversity of older immigrants will most likely continue to occur, governing bodies, program administrations, and academic leaders should too shift their gaze towards the unique set of barriers that affect distinct ethnocultural groups (Torres, 2006, as cited in Zubair \& Norris, 2015). Gaining this knowledge will not only prepare the Canadian immigrant system to adequately support an increasingly diverse and aging immigrant population, but will also ensure that these newcomers feel valued, supported and guided through a challenging transition in the later stages of life.

The first objective of this research paper was achieved by comparing the socioeconomic and ethnocultural characteristics of older immigrants with older non-immigrants. The purpose of this analysis is to understand the varying severity of economic disadvantage, racialization and marginalization that older immigrants face, in comparison to their Canadian-born counterparts. This objective is achieved by examining a series of cross-tabulations of select socioeconomic and ethnocultural variables for both population groups. Findings align with the literature, suggesting that older immigrants hold the substantial share of the older population in Toronto (Ng, 2012; Statistics Canada, 2011; Turcotte, 2007). An astounding $71.3 \%$ of all individuals aged 65 and above in the Toronto CMA are foreign-born, while only $28.7 \%$ are Canadian-born (Statistics Canada, 2018d). 
Demographic compositions reveal that age group and gender proportions are relatively comparable for both older immigrants and non-immigrant groups, with females making up the slightly larger share of each population, supported by relevant literature on age and gender. The inequity in gender distribution might be the result of unequal gender ratios at birth, mortality rates and life expectancy (Ritchie \& Roser, 2019). On average, women outlive men, resulting in a disproportionately higher population of females in any given population. Additionally, life expectancy of individuals from developing nations is lower than that of Canadians. Despite a slightly larger proportion of immigrants that are 75+ being observed, these findings contradict traditional life expectancies for developing nations (Ritchie \& Roser, 2019; World Health Organization, 2002). This shift could be attributed to the global trend of increasing life expectancies. More strikingly, as a result of imported labour resources in traditionally male-dominated industries, our findings could have revealed that males account for slightly more than half of the population, however, that was not conclusive (Ritchie \& Roser, 2019).

Pendakur and Pendakur (2011), Creese (2007) and Galabuzi (2006) contend that there are three factors which influence immigrant earnings: devaluing foreign labour, lack of knowledge of an official language, and fewer low-wage employment opportunities (as cited in Lo \& Wang, 2004). Thus, the research reinforces the notion that income, education, and language are intersecting elements that can make or break an older immigrant's successful adjustment and integration into Canadian society. Economically, older immigrants experience higher rates of depressed income than their Canadian-born counterparts which aligns with relevant literature (Aging in poverty in Canada, 2005; Dempsey, 2005; Government of Canada, 2018; Lo \& Wang, 2004; Mandell et al., 2018; Mental Health Commission of Canada, 2019; Ng, 2012; Picot \& Lu, 2017; Um \& Lightman, 2016). However, both subgroups exhibit proportionately higher observations of low income relative to the medium or high classes, suggesting that age, regardless of ethnocultural origins or immigrant status, influences depressed income for these subgroups. As presumed, this research reveals that immigrant status likely influences income depression, as approximately $80 \%$ of the older immigrants assessed in this research earn below 2017's Ontario average of was $\$ 42,500.00$ (Statistics Canada, 2019). These findings are also supported by Picot and Lu (2017) who concluded that the highest chronic low income rates in Canada, were observed in older immigrants (about $30 \%$ of all older immigrants) in contrast to older non-immigrants (about $2 \%$ ) (Ng, Lai, Rudner and Orpana, 2012). The prevalence of low income earnings in the older immigrant subgroups could be attributed to any one, or a combination of the influencing factors of devaluation of foreign labour, knowledge of English or French and fewer low-wage jobs.

Preston et al. (2010) and Picot et al. (2007) found that immigrants with higher levels of education tend to avoid chronic low income, but that a recent rise in immigrant education achievement has been echoed by a decline in income. Interestingly, this research paper demonstrated that despite $42 \%$ of older 
immigrants having a post-secondary degree, they consistently do not perform as well as their Canadianborn counterparts in income generation. The income gap present between highly educated older immigrants and non-immigrants exemplifies one of the three factors of income influence that Pendakur et al. (2011), discussed. Highly educated older immigrants exhibit significantly higher proportions of low income and significantly lower proportions of high income than older non-immigrants. Canada's labour market's perception of foreign labour and foreign-achieved education devalues an immigrant's qualification which is supported in relevant literature Durst and MacLean's (2010) research into diversity and aging among older immigrants in Canada (Frank \& Hou, 2017; Grant, 2005; Guo, 2009; Li, 2001; Osaze, 2017).

A high prevalence of no education is exhibited in the older immigrant population (34\%), compared to older non-immigrant (19\%) which could be attributed to a struggling education system in an immigrant's sending-country. This $34 \%$ is most likely composed predominantly of recent older immigrants, as long-standing older immigrants that arrived early enough to be enrolled in elementary school would have entered the Canadian education system. Overall, this research paper reveals devaluing of foreign labour as a result of education attainment is an influential factor in older immigrant earnings (Lo \& Wang, 2004).

Further to Pendakur and Pendakur's (2011), Creese's (2007) and Galabuzi's (2006) research, lack of an official Canadian language was found to significantly depress older immigrant earnings. Recent older immigrants, in particular, are not as fluent in official languages as their long-standing counterparts. Hence, a dependency on intergenerational support to navigate social services, public transportation and the labour market is expected (Dempsey, 2005; Lo \& Wang, 2004; Mandell et al., 2018). Consequently, this reliance decreases older immigrant independence and agency, further challenging their incomegenerating feasibility, as lack of knowledge of an official language reduces the ability to get steady employment. Despite the Federal government's implementation of communication and language programs for immigrants in 1992, they did not providing services that are ethnoculturally relevant, thus perpetuating the challenges in learning English or French (Guo, 2013; Laher, 2017; Mandell et al., 2018). Moreover, opportunities to learn and practice basic communication in one of Canada's official languages did not result in a decline of discrimination against immigrant labour (Creese, 2010). English and French speaking immigrants who retained an accent were still ostracized and faced challenges navigating the labour force, which depresses their ability to earn a living wage (Creese, 2010). Unfortunately, this points to the systemic issue of racialization in labour markets in general, which is out of the scope of this research paper.

Concurrently, the three factors which influence immigrant earnings (as noted by Pendakur and Pendakur (2011), Creese (2007) and Galabuzi (2006)) intersect with settlement location decisions that all 
immigrants make when leaving their home country (McDonald, 2004). Historically, immigrants of a particular ethnocultural group tend to settle in enclaves of co-ethnics communities to maintain cultural traditions and associations (Hiebert, 2015). Literature claims that recent immigrants are drawn to longstanding co-ethnic enclaves while long-standing immigrants are more economically independent and have the ability to resettle in other parts of the CMA (Hiebert, 2015; Vezina \& Houle, 2017). However, as supported by McDonald's (2004) findings, this study reveals that there is a divide, in that some recent immigrants settle within long-standing communities, while others decide to settle in regions outside of their co-ethnic enclaves. This is evident through the clustering of statistically significant recent immigrant groups in regions of the CMA that were not settled by their long-standing counterparts. Availability of low-wage employment, affordable housing, and intergenerational living arrangements might influence this distribution, considering that long-standing immigrants are more economically independent and could have resettled into more affluent neighbourhoods. Relative to the geography of the Toronto CMA, this research paper demonstrates that settlement patterns of long-standing older immigrants are found to be distributed throughout the Toronto CMA. In particular, this occurs within inner urban and suburban communities, in a linear pattern, north of Highway 401 and up through parts of Vaughan, Richmond Hill, and Markham. Select regions in Mississauga and some rural communities in peripheral municipalities also found older long-standing immigrants. However, this pattern could be the result of resettlement, which is a limitation as this analysis did not account for immigrant resettlement (Murdie, 2008). In contrast, recent older immigrants are observed to settle directly into the suburban regions. In some cases, this means settling in existing cluster of immigrants, like that of the Markham cluster, and in other cases untouched regions, like that of the Brampton-Mississauga cluster of recent older immigrants. This pattern of long-standing older immigrants settling into predominantly inner urban regions while their recent counterparts settle directly into suburban regions is supported by findings from Murdie (2008) and Vezina and Houle (2017).

\subsubsection{Variation in settlement patterns of long-standing and recent older immigrants}

Subsequent to socioeconomic, ethnocultural characteristics, and settlement patterns of the greater older immigrant population, this paper created a framework to identify the settlement variations between recent and long-standing older immigrants of ethnocultural-specific groups. The aforementioned discussion on socioeconomic and ethnocultural characteristics addresses the problematic perception that older immigrants are a homogeneous population and presents findings on where they are located.

The preceding discussion will examine the socioeconomic and ethnocultural characteristics and residential patterns of four select ethnocultural subgroups. This approach identifies clusters of recent (and long-standing) older immigrant group of Chinese, Italian, Portuguese and South Asian origins that likely 
require unique settlement services and information access as a result of their varying ethnocultural identities. This is particularly important given older immigrant's proclivity to experience chronic low income, inadequate and unaffordable housing, and limited educational opportunities, reduced access to social, health and settlement services, and compounded barriers to settlement and integration.

By examining a series of cross-tabulations of select socioeconomic and ethnocultural variables for the four ethnocultural subgroups and mapping the residential hot spots of each subgroup, categorized by immigration period the first objective of this research paper is achieved. In terms of age composition, South Asian older immigrants have the highest share of their older population aged 65-74 (64.3\%), while Italians have the lowest (47.7\%). Italians are the only group where individuals aged $75+$ account for slightly more than half of their older immigrant population, which can most likely be accounted for by their long-term aging in Canada, and overall longer life-expectancy (Ho \& Hendi, 2018; Lallo \& Raitano, 2018). All ethnocultural subgroups have slightly more than $50 \%$ female composition which aligns with traditional gender ratios.

Long-standing ethnocultural groups (Italian and Portuguese) are found to consistently have the highest rates of medium and high income, while their recent ethnocultural counterparts (Chinese and South Asian) had higher rates of low income. A possible explanation for these results may be attributed to homeland cultural importance assigned to property ownership, their longer opportunity to learn an official language over the course of their residency, and their skills in trades-related fields (Heibert, 2015; Murdie, 2008). Immigrants from southern Europe were typically employed in construction labour and were able to purchase inexpensive properties and renovate them (Murdie, 2008). Renovations would increase property values or could be pivoted towards construction of income property suites to subsidize their mortgage (Murdie, 2008). Thus, having more disposable income and the opportunity to sell and resettle into larger suburban or rural properties, which influences their spatial distribution (Heibert, 2015).

Recent subgroups (Chinese and South Asian) have higher proportions of secondary and postsecondary education, while long-standing subgroups (Italian and Portuguese) did not. South Asian older immigrants are the most fluent (33\%) in the official languages of all the ethnocultural subgroups, followed by Italians, and Portuguese. The high proportion of language proficiency for South Asians is likely a result of colonialism in the late 1600s, which saw bilingualism grow due to British occupation of South Asian nations (Fishman, 1978; Hickey, 2004). Chinese older immigrants have the highest share of non-official language speaking individuals (83\%) which might be attributed to the numerous dialects and within China's geography, which would require language-specific settlement services available in Canada (Lo \& Wang, 2004). Historically, Chinese have employed mitigation strategies to reduce the impact of cultural assimilation from British and American occupation, which were so effective that in the sixteenth and seventeenth centuries Westerners learnt spoken and written Chinese as per the then Emperor's 
directives (Adamson, 2004; Chan, 2011). Additionally, Chinese enclaves are visibly the largest of all subgroups examined, with concentrations located in both the downtown Toronto core and in suburban regions of the CMA. These ethnocultural enclaves are concentrated and well established and might have experienced commercial, residential and cultural revitalization as a result of the ethnic concentration, hence the ability for older immigrants with no knowledge of an official language to still flourish and integrate (Murdie, 2008). Unfortunately, the comfort, convenience and accessibility provided to older Chinese immigrants that settle within these large clusters could conserve cultural exclusiveness and act as barriers to adjustment and integration (Chan, 2012).

Additionally, long-standing immigrants perform better in income classes in almost all language categories, with the largest income gaps in the non-official language category, which might be explained by Murdie's (2008) findings of long-standing immigrant's construction background real estate prowess. Knowledge of an official language does not guarantee higher earners, as English and French speaking Chinese and South Asian older immigrants are proportionately higher in the low income class. This could be the result of discrimination in the labour force, as the Chinese and South Asian subgroups would be visible minorities that could experience racialization.

Regarding residential patterns of long-standing and recent older immigrants for each ethnocultural subgroup, this research paper determines that each ethnocultural subgroups exhibit different patterns of spatial mismatch. Overall, these findings echo those published by the University of Toronto, in that long-standing older immigrants (arriving before 1971), were observed to have settled in small clusters, adjacent to the downtown Toronto core as well as in inner urban suburbs along the VaughanToronto border, and that these immigrant groups were typically comprised of Greeks, Italians, and Portuguese (Fong \& Wilkes, 2003; Murdie, 2008). The settlement distribution of Italian and Portuguese older immigrants in this research paper are in support of Murdie's (2008) and were typically found to reside in inner urban regions (like Little Italy and Little Portugal), as well as in inner suburban regions, like along the Vaughan-Toronto border. Conversely, literature did not conclude that long-standing older Italian immigrants also had proportionality high residential uptake in most of Vaughan and parts of Caledon and King. This study was unable to demonstrate that, at large, recent and long-standing immigrants are found to not necessarily settle in clusters within the urban centres, but are distributed across the CMA in regions on the peripheral of the urban boundaries (Vezina \& Houle, 2017). In terms of spatial overlap, this research paper found that the recent older immigrant Italian and Portuguese subgroups appear to have the highest spatial mismatch in residential clustering with their long-standing counterparts. The recent subgroups appear to settle in regions outside of their long-standing counterpart clusters, in many instances, which might be explained by intergenerational living opportunities in that an older immigrant might be settling in a relative's home that is located outside of their ethnocultural 
enclave. More realistically, given this subgroup's proclivity in construction and renovations, they most likely resettled into more affluent areas and could sponsor additional family members from their country of origin. This shift might also be the result of processes of gentrification in downtown Toronto neighbourhoods that were typically receiving communities for immigrants, but the effects of gentrification and employment opportunities pushed them to settle elsewhere (Murdie, 2008).

The Chinese subgroups appear to have the highest instance of spatial overlap, where recent older immigrant settlement clusters are located within long-standing older Chinese immigrant clusters. Similarly, recent South Asian older immigrants exhibit spatial overlap on clusters of their long-standing counterparts. Higher concentrations of the recent subgroup cluster within one of the long-standing clusters, while recent South Asian clusters have travelled north, out of Toronto and into Markham and Richmond Hill. This shift is most likely due to the availability of lower-waged jobs and affordable housing outside of the inner city regions and into the suburban regions. Findings from Vezina and Houle (2017) that did correspond to patterns revealed in this research paper are that these suburban recent immigrants were found to have clustered in specific CTs in both the urban and suburban landscapes.

\subsection{Implications and recommendations}

Coupling the global trend of aging populations with Toronto's proclivity to attract immigrants of widening age-groups and ethnocultural origins, understanding the settlement patterns unique to ethnocultural-specific immigrant groups has become a central issue in immigration studies and policy planning. Key policy and program administrators should prioritize a plan for the provision of ethnocultural-specific settlement services in the Toronto CMA, specifically in recent older immigrant enclaves. In particular, they should focus on communities of recent older immigrants that decided not to settle within their co-ethnocultural long-standing immigrant enclaves. These older immigrants are highly vulnerable and more susceptible to the oppression of settlement challenges as they are not embedded in established co-ethnic enclaves which could afford them an easier settlement experience. As South Asian and Chinese older immigrants are proportionately higher in recent immigration periods, they should be given special attention. Despite having higher levels of education, they are economically depressed which is likely to be a consequence of language barriers. As lack of knowledge of an official language is an influential factor in income earning potential services in basic communication for these two subgroups should be developed. Hence, a practical application is to determine the linguistic composition of the South Asian and Chinese subgroups and ensure that policy makers, community leaders and local governments develop and implement language-specific settlement services within recent older South Asian and Chinese immigrant clusters. 


\subsection{Contribution to knowledge}

In response to Mandell, Borras and Phonepraseuth's (2018) call-to-action, this research documents the socioeconomic climate of older immigrants by landing period and identifies the differences in settlement patterns for four select ethnocultural groups based on their long-standing or recent immigration to Toronto. While there is limited scholarship on this subject, the findings from this study makes several contributions to the current literature. Firstly, it explores the nuanced differences in population composition of four ethnocultural-specific subgroups representing long-standing and recent immigrants and secondly, it identifies clusters of recent immigrants that are settling outside of their long-standing ethnocultural enclaves.

This research paper provides an important opportunity to advance the understanding of ethnocultural-specific older immigrant settlement patterns and socioeconomic status with hopes to facilitate better social, economic and cultural adjustment and integration into the Canadian society (Mandell et al., 2018). As the study populations in this research paper represent three levels of social marginalization (ethnocultural minorities, immigrants and older adults), their lived experience is particularly unique and their economic vulnerability is considerably high (Torres, 2006; Warnes et al. 2000 as cited in Zubair \& Norris, 2015). Considering the most impactful settlement challenges relate to barriers to language, program administration and regional governments should better understand the ethnocultural and linguistic characteristics of their regional populations, particularly for the most recently immigrated older populations. Hence, facilitating and implementing culturally appropriate services should become a priority. To do that, they need to know where these groups are located.

This research paper successfully identifies the location of clusters of recent older immigrants of four ethnocultural subgroups in the Toronto CMA, whose populations would likely be receptive to receive culturally appropriate information on social, cultural and settlement services. Future research should continue this shift in examination of issues experienced by older adult immigrants from varying ethnocultural identities as the global geopolitical landscape continues to facilitate displacement and immigration

\subsection{Limitations and future research}

The small number problem is a prevalent concern in this research paper considering the relatively small number of individuals in the study populations. While many CTs contained low or zero values, it is unknown if this was the result of data suppression, missing data or an actual count of zero. Privacy concerns typically result in data suppression, especially for CTs with very low populations. Despite using the Census Tract as the unit of geography (as it presented clusters of older immigrants at a finer precision), future research could use a larger unit of measure to counter this concern. While there was 
limited scholarship to support methodological decisions made throughout this analysis, this research paper functions as an exploratory pilot to fill said gap and decisions were made based on relevant literature and data distributions. Additionally, Statistics Canada was unable to comment on the nature of the income variables they provided, thus, this analysis assumed it was average individual income. Regarding methodological limitations, the recent older immigrant subgroup hot spot maps should be reviewed with caution as their study population was significantly smaller than the sample size of the longstanding subgroups.

Lastly, future research could include an age at immigration attribute in the cross-tabulations, which might skew the results differently to facilitate better cross-group comparisons. Examining older long-standing immigrants that landed as individuals aged 65 or above and navigated settlement in a pre2000 era compared to recently landed older immigrants would likely produce less result variations.

Researchers have not examined ethnocultural-specific recently landed older immigrant settlement analysis in much detail. Most studies focused on recent immigrants, older immigrants or recent older immigrants as a holistic group, neglecting to examine in-group differences based on ethnocultural origins. Hence, future research should continue to seek to understand the spatial and aspatial differences in older, ethnocultural-specific immigrants with hopes to foster an easier transition into Canadian society that occurs for these individuals at such a late stage in life. A full discussion of recent older immigrant employment in the Canadian labour market and how systemic and personal discrimination might influence employment rates of recent older immigrants should also be explored. Moreover, primary data, such as interviews with older immigrants from each of the ethnocultural subgroups would illuminate their individual story at a granular level. This would allow for better understanding of the totality of circumstances under which an older immigrant decided to migrate in, answering questions on their net worth once they arrived in Canada, the family structure they left behind, the order of their arrival within intergenerational immigration and the unique experiences the influenced their migration. As the global geopolitical landscape continues to ebb and flow, Canadian policy and program makers should shift their gaze towards understudied ethnoculturally-specific immigrant populations to inform policy and ultimately keep pace with ever-changing human migration patterns.

It is evident in this research that systemic and institutional racism underpinning pre-1967 immigrant policy inherently depressed the current socioeconomic outcomes of racialized older immigrants, and directly influenced their settlement patterns. This is particularly the case for recent older immigrants. As ethnocultural heterogeneity in the Toronto CMA will remain high in the future, such diversity should continue to inform immigration and settlement policy and residential develop considerations. This paper successfully identified the ethnocultural enclaves that are housing Toronto CMA's newest, oldest and most vulnerable immigrants, therefore settlement services should be employed 
in age and ethnoculturally appropriate formats to ease the adjustment and integration these older immigrants are experiencing as they settle into Canadian society. 


\section{References}

Adamson, B. (2004). Chinas English: a history of English in Chinese education (pp. 479). Hong Kong: Hong Kong University Press.

Albridge, H. (2017). How do we Measure Poverty? How Do We Measure Poverty? (May), 30-46.

Andresen, M. A. (2007). Location quotients, ambient populations, and the spatial analysis of crime in Vancouver, Canada. Environment and Planning A, 39(10), 2423-2444. doi:10.1068/a38187

Anselin, L. (1995). Local indicators of spatial organization -LISA. Research, 27(2), 1-25.

Asthana, S., Gibson, A., Moon, G., Brigham, P., \& Dicker, J. (2004). The demographic and social class basis of inequality in self-reported morbidity: An exploration using the Health Survey for England. Journal of Epidemiology and Community Health, 58(4), 303-307. https://doi.org/10.1136/jech.2002.003475

Biase, S.D., \& Bauder, H. (2005). Immigrant Settlement in Ontario: Location and Local Labour Markets. Canadian Ethnic Studies; Calgary 37(3): 114-135.

Chan, A. (2011). The Chinese in Toronto from 1878: From Outside to Inside the Circle (pp. 186). Dundurn Press, Ontario.

Chan, A. (2012). From Chinatown to Ethnoburb: The Chinese in Toronto. World Confederation of Institutes and Libraries in Chinese Overseas Studies, 1-11.

Chow, H. P. H. (2010). Growing old in Canada: Physical and psychological well-being among elderly Chinese immigrants. Ethnicity and Health, 15(1), 61-72. https://doi.org/10.1080/13557850903418810

Chui T, Tran K, Maheux H. (2006). Immigration in Canada: A portrait of the foreign-born population, 2006 Census. In Statistics Canada. https://doi.org/97-557-XIE

Chui, T., Anderson, T., Flanders, J. (2018). National Household Survey. Immigration and Ethnocultural Diversity in Canada, 99-010-X20(99), 1-23. In Statistics Canada. https://doi.org/99-010-X2011001

Creese, G. (2007). Racializing work/reproducing white privilege. In V. Shalla and W. Clement (Eds.) Work in tumultuous times: Critical perspectives, 192- 226. Montreal \& Toronto: McGill-Queen's University Press

Creese, G. 2010. Erasing English language competency: African migrants in Vancouver, Canada. Journal of International Migration and Integration 11(3):295-313. Retrieved from doi:10.1007/s12134-010-0139-3 
Cromley, R. G., \& Hanink, D. M. (2012). Focal Location Quotients: Specification and Applications. Geographical Analysis, 44(4), 398-410. https://doi.org/10.1111/j.1538-4632.2012.00852.x

City of Toronto. (2017a). The Toronto Seniors Strategy: Strategy Highlights. Retrieved from https://www.toronto.ca/wp-content/uploads/2017/11/97e5-seniors-strategyhighlights.pdf

City of Toronto. (2017b). 2016 Census: Income Backgrounder. Retrieved from https://www.toronto.ca/w-content/uploads/2017/10/8f41-2016-Census-Backgrounder-Income.pdf

Dempsey, C. (2005). Elderly Immigrants in Canada: Income Sources and Self Sufficiency. Citizenship and Immigration Canada. Retrieved from http://www.cic.gc.ca/english/pdf/research-stats/elderly.pdf

Durst, D., \& MacLean, M. J. (2010). Diversity and aging among immigrant seniors in Canada: Changing faces and greying temples. Calgary: Detselig Enterprises.

Fishman, J. A. (1978). Advances in the Study of Societal Multilingualism (pp. 406). Mouton Publishers: The Hague.

Fong, E., \& Wilkes, R. (2003). Racial and ethnic residential patterns in Canada. Sociological Forum, 18(4), 577-602. doi:10.1023/B:SOFO.0000003004.78713.2e

Frank, K., \& Hou, F. (2017). Over-education and Life Satisfaction among Immigrant and Non-immigrant Workers in Canada. Analytical Studies Branch Research Paper Series, (11). Retrieved from http://www.statcan.gc.ca/pub/11f0019m/11f0019m2017393-eng.htm

Galabuzi, G.E. (2006). Canada's creeping economic apartheid: The economic segregation and social marginalization of racialized groups. Toronto, ON: Centre for Social Justice Foundation. http://www.socialjustice.org/index.php?page5canada-s-creepingeconomic-apartheid

Gallagher, E., Verena, M., \& Keefe, J. (2006). Age-Friendly Rural and Remote Communities: A Guide. Federal/Provincial/Territorial Ministers Responsible for Seniors. http://www.phac-aspc.gc.ca/seniors-aines/altformats/pdf/publications/public/healthysante/age_friendly_rural/AFRRC_en.pdf

Getis, A., \& Ord, J. K. (1992). The analysis of spatial association by use of distance statistics. Geographical Analysis, 24(3), 189-206. doi:10.1111/j.1538-4632.1992.tb00261.x

Government of Canada. (2005). Seniors on the margins Aging in poverty in Canada. Retrieved from http://publications.gc.ca/collections/Collection/H88-5-3-2005E.pdf

Government of Canada. (2018). Social isolation of seniors: a Focus on New Immigrant and Refugee Seniors in Canada. Retrieved from 
https://www.canada.ca/en/employment-social-development/corporate/seniors/forum/socialisolation-immigrant-refugee.html\#h2.2

Grant, P. R. (2005). The Devaluation of Immigrants' Foreign Credentials: The Psychological Impact of This Barrier To Integration into Canadian Society.

Guo, Lan. (2013). Language Policies and Programs for Adult Immigrants in Canada: A Critical Analysis. Canadian Ethnic Studies Journal 45(1-2):23-41. Retrieved from doi:10.1353/ces.2013.0022

Guruge, S., Thomson, M. S., \& Seifi, S. G. (2015). Mental Health and Service Issues Faced by Older Immigrants in Canada: A Scoping Review. Canadian Journal on Aging 34(4):431-444. Retrieved from doi:10.1017/S0714980815000379

Hickey, R. (2011). Legacies of colonial English: studies in transported dialects. Cambridge: Cambridge University Press.

Hiebert, D. (2015). Ethnocultural minority enclaves in Montreal, Toronto and Vancouver. IRPP Study, (52), 0-1.

Ho, J. Y., \& Hendi, A. S. (2018). Recent trends in life expectancy across high income countries: retrospective observational study. BMJ (Clinical research ed.), 362, k2562. doi:10.1136/bmj.k2562

Kang, Y., Cho, N., \& Son, S. (2018). Spatiotemporal characteristics of elderly population's traffic accidents in seoul using space-time cube and space-time kernel density estimation. PloS One, 13(5), e0196845-e0196845. doi:10.1371/journal.pone.0196845

King, K. M. (2009). The Geography of Immigration in Canada: Settlement, Education, Labour Activity And Occupation Profiles Working Paper Series: Ontario in the Creative Age The Geography of Immigration in Canada: Settlement, Education, Labour Activity and Occupation Profiles. (March).

Laher, N. (2017). Diversity, Aging, and Intersectionality in Ontario Home Care. (May). Retrieved from www.wellesleyinstitute.com.

Lai, D.W. L. (2000a). Depression among the elderly Chinese in Canada. Canadian Journal on Aging, 19(3), 409-429. Retrieved from http://ezproxy.lib.ryerson.ca/login?url=https://search-proquestcom.ezproxy.lib.ryerson.ca/docview/231980754?accountid=13631

Lai, D. W. L. (2000b). Prevalence of depression among the elderly Chinese in Canada. Canadian Journal of Public Health, 91(1), 64-6. Retrieved from http://ezproxy.lib.ryerson.ca/login?url=https://search-proquestcom.ezproxy.lib.ryerson.ca/docview/231980754?accountid=13631 
Lai, D.W. L. (2003). Health and predictors of health among older Chinese-Canadians in British Columbia. BC Medical Journal, 45(8), 382-390.

Lai, D., \& Yuen, C. (2003). Gender, physical limitation and depression among elderly Chinese. ECOMMUNITY: International Journal of Mental Health \& Addiction, 1(1), 1-16. https://doi.org/10.11575/PRISM/9622

Lallo, C. \& Raitano, M. (2018). Life expectancy inequalities in the elderly by socioeconomic status: evidence from Italy. Population Health Metrics 16(7). https://doi.org/10.1186/s12963-018-01637

Lambert D.M., Wilcox M.D., Clark C.D., Murphy B., Park W.M. (2010) Is Growth in the Health Sector Correlated with Later-Life Migration? In: Páez A., Gallo J., Buliung R., Dall'erba S. (eds) Progress in Spatial Analysis. Advances in Spatial Science (The Regional Science Series). Springer, Berlin, Heidelberg

Li, P. S. (2001). The market worth of immigrants' educational credentials. Canadian Public Policy / Analyse De Politiques, 27(1), 23-38. doi:10.2307/3552371

Lo, L., \& Wang, L. (2004). A political economy approach to understanding the economic incorporation of chinese sub-ethnic groups. Journal of International Migration and Integration / Revue De l'Integration Et De La Migration Internationale, 5(1), 107-140. doi:10.1007/s12134-004-1004-z

Malczewski, J. (2010). Exploring spatial autocorrelation of life expectancy in poland with global and local statistics. Geojournal, 75(1), 79-92. doi:10.1007/s10708-009-9278-5

Malenfant, É. C., Lebel, A., Martel, L., \& Statistics Canada. Demography Division. (2010). Projections of the diversity of the canadian population, 2006-2031 Statistics Canada, Demography Division.

Mandell, N., Borras, J., \& Phonepraseuth, J. (2018). Recent Canadian Immigrant Seniors: A Literature Review of Settlement Experiences and Services Knowledge Synthesis Report A Research and Knowledge Mobilization Project on the Settlement Outcomes-Services Nexus.

Mandell, N., Lam, L., Borras. J., \& Phonepraseuth, J. (2018). Living on the Margins: Economic Security Among Senior Immigrants in Canada 38-64.

Matheson, F.I. (2016). Ontario Agency for Health Protection and Promotion (Public Health Ontario). 2016 Ontario marginalization index. Toronto, ON: St. Michael's Hospital.

Mathews, R. (2000). Cultural Patterns of South Asian and Southeast Asian Americans. Intervention in School and Clinic, 36(2), 101-104. https://doi.org/10.1177/105345120003600205

Mazzulla, G., \& Forciniti, C. (2012). Spatial association techniques for analysing trip distribution in an 
urban area. European Transport Research Review, 4(4), 217-233. doi:10.1007/s12544-012-0082-

McDonald, L. (2001). A study on the settlement related needs of newly arrived immigrant seniors in Ontario University of Toronto, Faculty of Social Work.

McDonald, J. T. (2004). Toronto and Vancouver bound: The location choice of new Canadian immigrants.

Canadian Journal of Urban Research, 13(1), 85-101

Mental Health Commission of Canada. (2019). Immigrant, refugee, ethnocultural and racialized populations and the social determinants of health. A review of 2016 census data https://www.mentalhealthcommission.ca/sites/default/files/201903/irer_report mar 2019 eng.pdf

Murdie, R. A., \& University of Toronto. Cities Centre. (2008). Diversity and concentration in Canadian immigration: Trends in Toronto, Montréal and Vancouver, 1971-2006 Centre for Urban \& Community Studies.

National Institute on Aging and National Institute of Health. (2011). Global Health and Aging. World Health Organization. Retrieved from https://www.who.int/ageing/publications/global_health.pdf?ua=1

Nazroo, J. Y. (2006). Ethnicity and old age In Vincent J. A. The Futures of Old Age. Sage, London, 62 72

Ng, E., Lai, D. W. L., Rudner, A. T., Orpana, H., \& Ali, M. A. (2012). What do we know about immigrant seniors aging in Canada? A demographic, socio-economic and health profile CERIS - The Ontario Metropolis Centre.

Ng, E., Sanmartin, C., Tu, J., \& Manuel, D. (2014). Use of acute care hospital services by immigrant seniors in Ontario: A linkage study. Health Reports, 25(10), 15-22.

Osaze, E. (2017) The Non-Recognition or Devaluation of Foreign Professional Immigrant's Credentials in Canada: The Impact on the Receiving Country (Canada) and the Immigrants. Retrieved from https://yorkspace.library.yorku.ca/xmlui/bitstream/handle/10315/34314/Osaze_Emmanuel_Dean_ 2017_MA.pdf?sequence $=2 \&$ isAllowed $=y$

Pendakur, K., \& Pendakur, R. (2011). Color by numbers: Minority earnings in Canada 1995-2005. Journal of International Migration and Integration, 12(3), 305-329. doi:10.1007/s12134-0100160-6

Phillipson C., Alhaq E., Ullah S. and Ogg, J. (2000). Bangladeshi families in Bethnal Green, London: older people, ethnicity and social exclusion In Warnes A. M., Warren L. and Nolan M. (eds), Care Services for Later Life: Transformations and Critiques. Jessica Kingsley, London, 273-90. 
Picot, G., \& Feng, H. (2014). Analytical studies branch research paper series - Immigration, low income and income inequality in Canada: What's new in the 2000s. Retrieved from http://www.statcan.gc.ca/pub/11f0019m/11f0019m2014364-eng.pdf

Picot, W. G., Coulombe, S., Hou, F., Statistics Canada. Analytical Studies Branch, \& Statistics Canada. Business and Labour Market Analysis Division. (2007). Chronic low income and low income dynamics among recent immigrants Statistics Canada, Analytical Studies Branch.

Preston, V. A., \& Toronto Immigrant Employment Data Initiative (TIEDI). (2010). What are the labour market outcomes for university-educated immigrants? Toronto Immigrant Employment Data Initiative (TIEDI).

Ritchie, H., \& Roser, M. (2019), Gender Ratio. Our World in Data. Retrieved from https://ourworldindata.org/gender-ratio

Sadavoy, J., Meier, R., \& Ong, A. Y. M. (2004). Barriers to access to mental health services for ethnic seniors: The Toronto study. The Canadian Journal of Psychiatry, 49(3), 192-199. doi:10.1177/070674370404900307

Séguin, A., Apparicio, P., Riva, M. \& Negron-Poblete, P. (2016) The Changing Spatial Distribution of Montreal Seniors at the Neighbourhood Level: A Trajectory Analysis, Housing Studies, 31:1, 6180, DOI: 10.1080/02673037.2015.1061106

Simich, L., \& Maytree Foundation. (2000). Towards a greater Toronto charter: Implications for immigrant settlement The Maytree Foundation.

Somenahalli, S. V. C., Shipton, M. D., \& Bruce, D. (2010). Investigating the spatial distribution of the elderly and its implications for service provision in Adelaide Metropolitan Area. ATRF 2010: 33rd Australasian Transport Research Forum, (October), 1-16.

Statistics Canada. (2006). Census of Population (Catalogue 94-557-XCB2006021) Ottawa: Statistics Canada.

Statistics Canada. (2007). Immigration and citizenship. 2006 Census release topics. Ottawa: Statistics Canada.

Statistics Canada. (2009). Population Projections for Canada, Provinces and Territories, 2009-2036 (Catalogue 91-520-X). Ottawa: Statistics Canada.

Statistics Canada. (2012a). Low Income Lines, 2010 to 2011. Income Research Paper Series, (75), 1-38. Retrieved from http://www.statcan.gc.ca/pub/75f0002m/75f0002m2012002-eng.pdf

Statistics Canada. (2012b). The Canadian Population in 2011: Age and Sex (Catalogue 
98-311-X2011001). Ottawa: Statistics Canada.

Statistics Canada. (2013). 2011 National Household Survey: Immigration, place of birth, citizenship, ethnic origin, visible minorities, language and religion. The Daily Catalogue No. 11-001-X, 2011-2014.

Statistics Canada. (2016a). Census Profile, 2016 Census. Ottawa: Statistics Canada.

Statistics Canada. (2016). 150 years of immigration to Canada. Ottawa: Statistics Canada. Retrieved from https://www150.statcan.gc.ca/n1/pub/11-630-x/11-630-x2016006-eng.htm

Statistics Canada. (2018a). Annual Demographic Estimates: Sub provincial Areas, Census metropolitan areas. Ottawa: Statistics Canada. Retrieved from https://www150.statcan.gc.ca/n1/pub/91-214x/2018000/section01-eng.htm

Statistics Canada. (2018b). Immigration and Ethnocultural Diversity in Canada. Ottawa: Statistics Canada. Retrieved from https://www12.statcan.gc.ca/nhs-enm/2011/as-sa/99-010-x/99-010-x2011001-eng.cfm

Statistics Canada. (2018c.) Census Tracts: Detailed Definition. Ottawa: Statistics Canada. Retrieved from https://www150.statcan.gc.ca/n1/pub/92-195-x/2011001/geo/ct-sr/def-eng.htm

Statistics Canada. (2018d). Custom 2016 Census data of population aged 15 years and above in private households. Unpublished raw data.

Stewart, M., Shizha, E., Makwarimba, E., Spitzer, D., Khalema, E. N., \& Nsaliwa, C. D. (2011). Challenges and barriers to services for immigrant seniors in Canada: "you are among others but you feel alone". International Journal of Migration, Health and Social Care, 7(1), 16-32. doi:10.1108/17479891111176278

Taylor, L. E., Taylor-Henley, S., \& Doan, L. (2005). Older immigrants: Language competencies and Mental health. Canadian Journal of Community Mental Health $=$ Revue Canadienne De Sante Mentale Communautaire, 24(2), 23-34. doi:10.7870/cjcmh-2005-0012

Torres, S., Mälardalens högskola, \& Institutionen för samhälls- och beteendevetenskap. (2006). Elderly immigrants in Sweden: 'otherness' under construction. Journal of Ethnic and Migration Studies, 32(8), 1341-1358. doi:10.1080/13691830600928730

Turcotte, M., Schellenberg, G., \& Statistics Canada. Social and Aboriginal Statistics Division. (2007). A portrait of seniors in Canada, 2006 Statistics Canada, Social and Aboriginal Statistics Division.

Um, S., \& Lightman, N. (2016). Ensuring Healthy Aging for All Home Care Access for Diverse Senior. Retrieved from http://www.wellesleyinstitute.com/wp-content/uploads/2016/07/EnsuringHealthy-Aging-For-All_Wellesley-Institute.pdf 
Varga, C., Pearl, D. L., McEwen, S. A., Sargeant, J. M., Pollari, F., \& Guerin, M. T. (2015). Area-level global and local clustering of human Salmonella enteritidis infection rates in the city of Toronto, Canada, 2007-2009. BMC infectious diseases, 15(1), 359.

Vézina, M., \& Houle, R. (2017). Settlement Patterns and Social Integration of the Population with an Immigrant Background in the Montréal, Toronto and Vancouver Metropolitan Areas. Retrieved from http://www.statcan.gc.ca/pub/89-657-x/89-657-x2016002-eng.pdf

Warnes, A. M., Friedrich, K., Kellaher, L. \& Torres, S. (2004). The diversity and welfare of older migrants in Europe. Ageing \& Society, 24(3), 307-26.

World Health Organization. (2002). Proposed working definition of an older person in Africa for the MDS Project. Health Statistics and Information Systems. Geneva: World Health Organization. Retrieved from https://www.who.int/healthinfo/survey/ageingdefnolder/en/

World Health Organization. (2014). World health statistics, 2014. Geneva: World Health Organization. Retrieved from https://www.who.int/gho/publications/world_health_statistics/2014/en/

Yamashita, T., \& Kunkel, S. R. (2012). Geographic access to healthy and unhealthy foods for the older population in a U.S. Metropolitan area. Journal of Applied Gerontology, 31(3), 287-313.

Yu, W. K. (2000). Chinese Older People: A Need for Social Inclusion in Two Communities. Policy Press, Bristol, UK.

Zubair, M., \& Norris, M. (2015). Perspectives on ageing, later life and ethnicity: Ageing research in ethnic minority contexts. Ageing and Society, 35(5), 897-916. doi:10.1017/S0144686X14001536 